Policy Research Working Paper 2811

\title{
The Epidemiological Impact of an HIV/AIDS Vaccine in Developing Countries
}

\author{
John Stover \\ Geoff P. Garnett \\ Steve Seitz \\ Steven Forsythe
}

The World Bank

Development Research Group

Public Services

March 2002 
Policy Research Working Paper 2811

\section{Abstract}

Many people see an effective preventive AIDS vaccine as the best solution to the HIV/AIDS pandemic. Ten years ago many scientists had hoped that a vaccine would be available by now. Most scientists are still optimistic that vaccines will be developed and many candidates are being tested. Strategies to implement HIV/AIDS vaccination need to be developed to be ready when vaccines do become available. The nature of those programs will depend on the characteristics of each vaccine. How much does it cost? How effective is it? How long does protection last? The answers to these and other questions will help determine issues such as: What will be the impact of the vaccine on the epidemic? Who should be vaccinated? Will an AIDS vaccine be more cost-effective than other prevention measures? Will other measures still be necessary? What will happen to the epidemic if vaccination leads to riskier behavior? How much funding will be needed?

Stover, Garnett, Seitz, and Forsythe use two computer simulation models to investigate the effects of various vaccine characteristics and implementation strategies on the impact and cost-effectiveness of vaccines in different contexts. A simulation model is applied to data from rural Zimbabwe and the iwgAIDS model is applied to Kampala (Uganda) and Thailand. The models are used to investigate the effects of efficacy, duration, cost, and type of protection on impact and cost-effectiveness. The models also show the merits of targeting public subsidies to various population groups: all adults, teenagers, high- risk groups, and women of reproductive age. The impact of vaccines on the epidemic is compared with the impact of other prevention interventions such as condom use and behavior change. Finally, the models are used to explore the extent with which behavioral reversals may erode the positive benefits of the vaccine.

A highly effective, long-lasting, inexpensive vaccine would be ideal and could make a major contribution in controlling the HIV/AIDS pandemic. But vaccines that do not attain this ideal can still be useful. A vaccine with 50 percent efficacy and 10 years duration supplied to 65 percent of all adults could reduce HIV incidence by 25 to 60 percent, depending on the context and stage of the epidemic. Better efficacy and longer duration would provide even more impact. Programs focused on teenagers or high-risk populations have less overall impact but would provide significant benefits at much less cost than those reaching all adults. Behavioral reversals could erode much of the benefits of vaccination programs so it will be important to combine vaccination with continued messages about the importance of safe behaviors.

The cost of the vaccines is not known at this time. At a cost of $\$ 10$ or $\$ 20$ per person vaccinated, the cost per infection averted would be as low or lower than other prevention interventions. Higher costs for the vaccines and the need for many booster shots could reduce the cost-effectiveness significantly.

This paper-a product of Public Services, Development Research Group-is part of a joint research project with the European Commission on "The Economics of AIDS Vaccines in Developing Countries." Copies of this paper are available free from the World Bank, 1818 H Street NW, Washington, DC 20433. Please contact Hedy Sladovich, mail stop MC3311, telephone 202-473-7698, fax 202-522-1154, email address hsladovich@ ${ }^{\prime}$ worldbank.org. Policy Research Working Papers are also posted on the Web at http://econ.worldbank.org. The authors may be contacted at j.stover@tfgi.com, g.garnett@ic.ac.uk, s-seitz@uiuc.edu, or s.forsythe@itfgi.com. March 2002. (30 pages)

The Policy Research Working Paper Series disseminates the findings of work in progress to encourage the exchange of ideas about development issues. An objective of the series is to get the findings out quickly, even if the presentations are less than fully polished. The papers carry the names of the authors and should be cited accordingly. The findings, interpretations, and conclusions expressed in this paper are entirely those of the authors. They do not necessarily represent the view of the World Bank, its Executive Directors, or the countries they represent. 


\title{
The Epidemiological Impact of an HIV/AIDS Vaccine in Developing Countries
}

\author{
John Stover \\ The Futures Group International \\ Dr. Geoff P. Garnett \\ Imperial College \\ Dr. Steve Seitz \\ University of Illinois \\ Dr. Steven Forsythe \\ The Futures Group International
}

This paper is a product of the research project on "The economics of an HIV/AIDS vaccine in developing countries: Potential impact, cost effectiveness and willingness to pay." sponsored by the European Commission and the Development Research Group of the World Bank (task manager, Martha Ainsworth). The project was launched in response to recommendations of the World Bank's AIDS Vaccine Task Force, issued in May 2000. The authors wish to acknowledge comments received on earlier drafts from Martha Ainsworth, Amie Batson, Eduard Bos, Michael Kremer, Yvette Madrid, Mead Over, and Jane Rowley. The opinions and conclusions expressed in this paper are those of the authors and do not necessarily reflect the position of the European Commission or the World Bank or its members. 



\section{Contents}

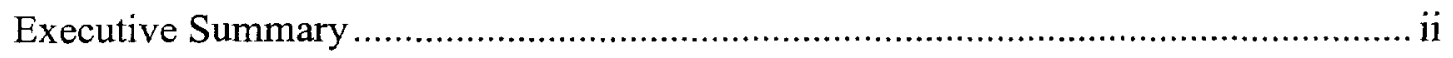

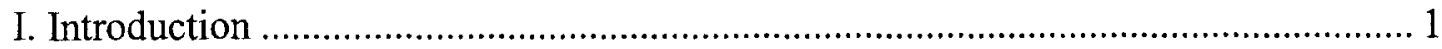

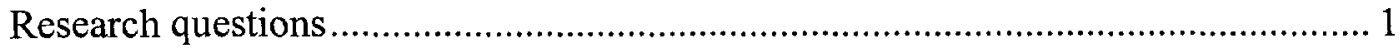

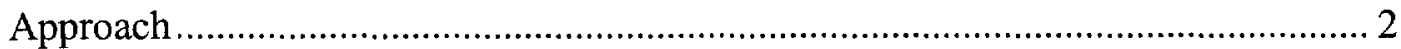

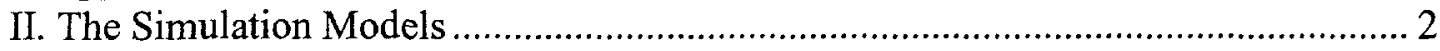

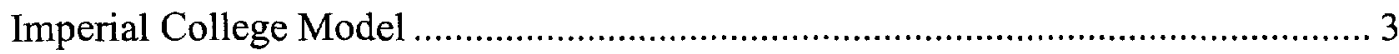

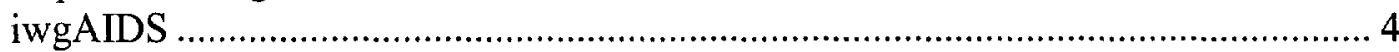

III. Descriptions of the epidemics analyzed ........................................................... 4

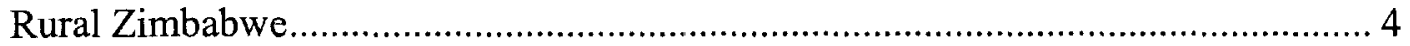

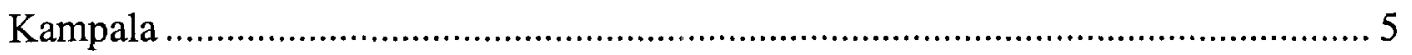

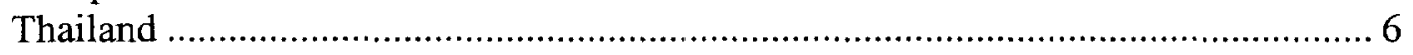

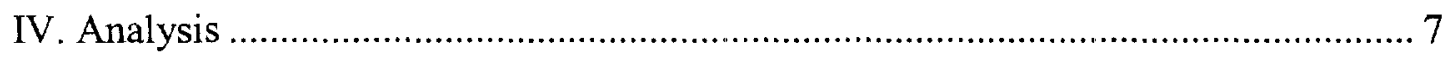

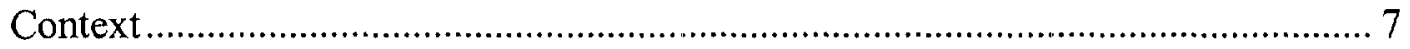

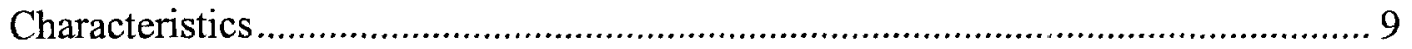

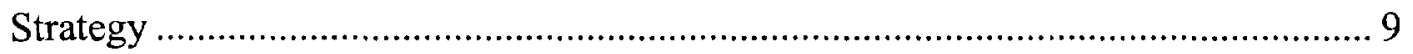

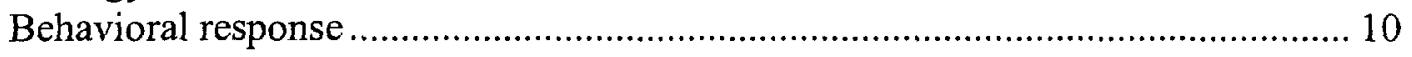

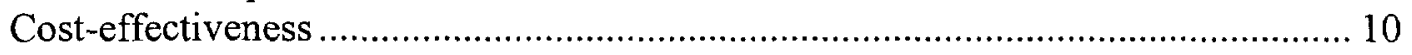

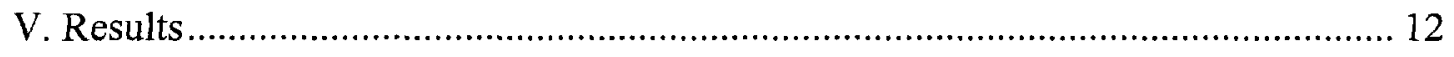

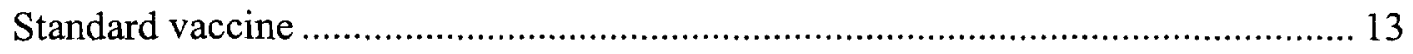

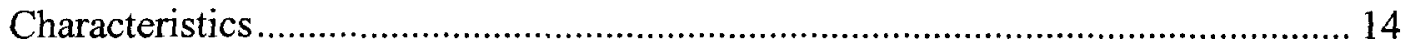

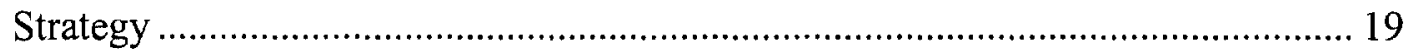

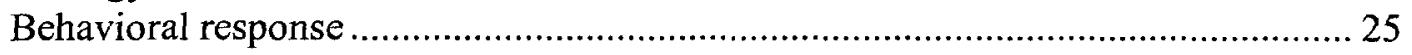

Comparison with other prevention interventions ....................................... 27

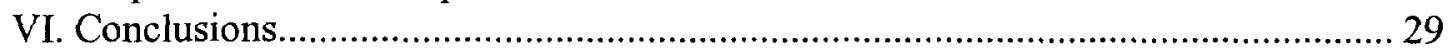




\section{Executive Summary}

Many people see an effective AIDS vaccine as the best solution to the HIV/AIDS pandemic. A considerable amount of funding and research effort is devoted to developing an effective vaccine. Ten years ago many scientists had hoped that a vaccine would be available by now. Most scientists are still optimistic that vaccines will be developed and many candidates are being tested. Programs to implement vaccination need to be developed in order to be ready when vaccines do become available. The nature of those programs will depend on the characteristics of each vaccine. How much does it cost? How effective is it? How long does protection last? The answers to these and other questions will help determine issues such as: Who should be vaccinated? Should regular re-vaccinations be scheduled? How much funding will be needed? Do vaccination campaigns need to be supported with safe sex messages? What will be the impact of the vaccine on the epidemic?

This study uses two computer simulation models to investigate the effects of various vaccine characteristics and implementation strategies on the impact and costeffectiveness of vaccines in different contexts. A simulation model from the Imperial College is applied to data from rural Zimbabwe and the iwgAIDS model is applied to Kampala and Thailand. The models are used to investigate the effects of efficacy, duration, cost and type of protection on impact and cost-effectiveness. The models also illustrate the merits of targeting public subsidies to various population groups: all adults, teenagers, high-risk groups and reproductive age women. The impact of vaccines on the epidemic is compared with the impact of other prevention interventions, such as condom use and behavior change. Finally, the models are used to explore the extent to which behavioral reversals may erode the positive benefits of the vaccine.

A highly effective, long-lasting, inexpensive vaccine would be ideal and could make a major contribution to controlling the HIV/AIDS pandemic. However, vaccines that do not attain this ideal can still be useful. A vaccine with 50 percent efficacy and 10 years duration supplied to 65 percent of all adults could reduce HIV incidence by 25 to 60 percent depending on the context and stage of the epidemic. Better efficacy and longer duration would provide even more impact. Programs focused on teenagers or high-risk populations have less overall impact but would provide significant benefits at much less cost than those reaching all adults. Behavioral reversals could erode much of the benefits of vaccination programs so it will be important to combine vaccination with continued messages about the importance of safe behaviors.

The cost of the vaccines is not known at this time. At a cost of $\$ 10$ or $\$ 20$ per person vaccinated the cost per infection averted would be as low or lower than other prevention interventions. Higher costs for the vaccines and the need for many booster shots could reduce the cost-effectiveness significantly. 


\section{Introduction}

Roughly 95 percent of the 34 million people living with HIV/AIDS globally are in developing countries. ${ }^{1}$ Nearly three-quarters of infections are in Sub-Saharan Africa, where in some countries more than a fifth of the adult population is infected. A number of preventive interventions have been found effective, either in pilot projects or national AIDS programs, in slowing the spread of infection-reduction in partners, increased condom use, sexually transmitted disease (STD) treatment, harm reduction programs, and use of drugs to reduce mother to child transmission. ${ }^{2}$ A preventive AIDS vaccine would greatly enhance the goal of containing the epidemic. However, the scientific challenges are great. More than 25 vaccine candidates have been developed but only one vaccine is currently in phase III clinical trials, and the full effectiveness of that vaccine, which requires 7 doses, will not be known until 2002-3. The first vaccines developed will most likely be expensive and less than fully effective. ${ }^{3}$

As the first preventive AIDS vaccines become available, policymakers charged with public health strategies to reduce the spread of HIV in the population need to understand (1) what vaccine characteristics, levels of coverage, and immunization strategies will have the largest impact on halting the spread of the epidemic; and (2) the effectiveness of an AIDS vaccine compared with other prevention interventions and strategies. ${ }^{4}$ A related issue is the extent to which vaccination may lead recipients to engage in riskier behavior, a 'behavioral reversal' that could result in heightened spread of infection with low efficacy vaccines. Such information is essential to develop a realistic and effective strategy for the use of an AIDS vaccine, the potential public health need for an AIDS vaccine and the potential demand (willingness to pay) of governments and international agencies for an AIDS vaccine of different characteristics for the poorest countries. Computer modeling has shown that even partially effective vaccines can have a substantial impact on the course of the epidemic, if coverage is high, but the full benefits may not be achieved in the poorest countries if the cost of producing and distributing the vaccine is high. ${ }^{5}$

\section{Research questions}

The objective of this study is to answer the following questions concerning the impact of a preventive AIDS vaccine for adults:

\footnotetext{
${ }^{1}$ UNAIDS. 2000. Report on the global HIV/AIDS epidemic, June 2000. Geneva: UNAIDS.

${ }^{2}$ Michael Merson, Julia Dayton, and Kevin O'Reilly. 2000. "Effectiveness of HIV prevention interventions in developing countries." AIDS 14 (suppl 2): S68-S84.

${ }^{3}$ World Bank AIDS Vaccine Task Force. 2000. "Accelerating an AIDS vaccine for developing countries: Recommendations for the World Bank." Washington, D.C., May.

${ }^{4}$ V. Tangcharoensathien, W. Phoolcharoen, S. Pitayarangsarit, S. Kongsin, V. Kasemsup, S. Tantivess and others. 2001. "The potential demand for an AIDS vaccine in Thailand." Health Policy 57(2):111-39.

${ }^{5}$ R. M. Anderson, J. Swintin, and G. P. Garnett. 1996 "Low-efficacy HIV vaccines: Potential for community-based intervention programs." Lancet $348: 1010-13$
} 
1. What is the impact of vaccine characteristics and immunization strategy on the spread of HIV in the population? If the initial vaccines are costly and only partially effective, for whom should the vaccine be provided to have the maximum impact on the epidemic?

2. Under what circumstances can behavioral reversals among those vaccinated cancel out the benefits of an AIDS immunization program?

3. How effective is an AIDS vaccine in stopping the spread of AIDS in the population compared to other targeted and untargeted prevention methods, such as reduction in sexual partners, increased condom use and improved treatment of sexually transmitted diseases (STD)? Are there thresholds in terms of vaccine efficacy or coverage that are likely to signal when an AIDS vaccine will become the dominant, most cost-effective strategy for preventing HIV/AIDS?

\section{Approach}

Two computer simulation models have been used to develop answers to these questions. Simulation modeling is a useful approach because it can illustrate the effects of vaccines that have not yet been developed. Vaccines with different characteristics can be tested to determine the effects of these characteristics on the outcomes. We have also used the models to test various implementation strategies and behavioral responses. The models provide great flexibility to examine a number of hypothetical situations. However, even if we focus on a limited set of characteristics, strategies and responses the number of potential simulations is large. For this reason, we have chosen a "standard" vaccine (described in detail below) that is compared against a number of alternatives. The number of different simulations is too large to describe them all in this report. Selected simulations are discussed in order to illustrate the conclusions that we have drawn from the much larger number of simulations performed.

The simulation models used in this study require a considerable amount of effort to apply to a new setting. For this reason, we have chosen to apply the models to different settings rather than try to apply both models to all three settings. Three different epidemic settings are used: Kampala (Uganda), rural Zimbabwe, and Thailand. The combination of two different models and teams simulating vaccine effects in three different settings provides considerable diversity. Conclusions that emerge from a consensus of this work should be robust and not dependent on a particular approach or set of initial conditions.

\section{The Simulation Models}

This study uses two simulation models to investigate the impact of AIDS vaccines: the Imperial College model developed by Geoff Garnett, Roy Anderson and colleagues at the Imperial College and iwgAIDS developed by Steve Seitz at the University of Illinois. Although the models share some characteristics, there are many differences as well. In addition to the differences in the models, there are differences in the way each modeling 
team approached the problem of simulating the effects of vaccines. These differences in models and approach have led to a greater exploration of the effects of vaccines and in our understanding of the significant dynamics than if we had used a single model. The conclusions in this study are supported by results from both models. Any differences in results between the models are explained by differences in model structure or approach. Greater detail on the structure and application of these models is available in the specific model reports that accompany this summary report. ${ }^{6,7}$

\section{Imperial College Model}

The Imperial College (IC) model is derived from a long tradition of HIV/AIDS models developed by Roy Anderson, Geoff Garnett and others now at the Imperial College in London. ${ }^{8,9}$ The version of the model used for this study examines the heterosexual spread of HIV in a population of adults aged 15-49. The population is segregated by age, sex, and sexual activity class. New entrants into the adult population (those aged 15) are assigned to one of four activity classes. Individuals stay in their assigned activity class for life, but the characteristics of the class vary with age.

The model further divides the population into four categories according to immunization status: (1) fully immunized, (2) partially immunized, (3) not immunized and (4) previously vaccinated but no longer protected by the vaccine and not yet eligible for revaccination. Once a person becomes infected, he or she progresses eventually to AIDS and death.

The effect of a vaccine in the model is to move the vaccinated person from the susceptible (not immunized) category to the fully immunized or partially immunized category, depending on the type of vaccine. A fully immunized person is completely protected from HIV infection. A partially immunized person has a reduced probability of HIV infection. If the duration of the protection of the vaccine is not lifetime, then a person can move from the fully or partially immunized category to the previously vaccinated category, where he or she is fully exposed to the risk of HIV infection.

\footnotetext{
${ }^{6}$ Geoff P. Garnett and John Williams. 2001. "The potential impact of prophylactic HIV vaccination as a function of vaccine properties.” Department of Infectious Disease Epidemiology, Imperial College, London, July.

7 Steve Seitz. 2001. "The Epidemiological Impact of an HIV/AIDS Vaccine in Developing Countries." Computational Modeling Laboratory, University of Illinois, Champagne-Urbana, July.

${ }^{8}$ Geoff P. Garnett and R.M. Anderson. 1993. "Factors controlling the spread of HIV in heterosexual communities: patterns of mixing between different age and sexual activity classes." Philosophical Transactions of the Royal Society, London B 342: 137-59.

${ }^{9}$ Geoff P. Garnett. 1998. "The influence of behavioral heterogeneity on the population level impact of potential prophylactic HIV-1 vaccines." Journal of the Royal Scientific Society Series A 161:209-25.
} 


\section{iwg AIDS}

The original development of the iwgAIDS model was supported by an interagency working group of the U.S. government. A team of scientists developed the initial model. ${ }^{10,11,12}$ The software was originally developed at the University of Illinois and for the past decade, it has been maintained and improved by Steve Seitz and colleagues at the University of Illinois. This model includes a large number of individual characteristics that can affect the probability of HIV transmission including age, sex, marital status, urban/rural residence, level of sexual activity, circumcision, presence of sexually transmitted diseases, condom use, perinatal transmission, homosexual contact, injecting drug use, etc. Individual characteristics are continuously distributed over age. As a result, risk can vary considerably from one age to the next and within age by marital status, sexual preference, etc.

In iwgAIDS individuals can be vaccinated with fully protecting or partially protecting vaccines. If vaccine protection wanes, individuals become eligible for re-vaccination as a function of the time since vaccination and the half-life of the vaccine.

\section{Descriptions of the epidemics analyzed}

The models have been used to simulate the effects of an AIDS vaccine in three different settings: rural Zimbabwe, Kampala and Thailand.

\section{Rural Zimbabwe}

The Imperial College model uses a data set from Manicaland in rural Zimbabwe to test the effects of vaccines. The simulations are based on an extensive data set collected over several years in rural communities in Manicaland. ${ }^{13}$ Most HIV transmission occurs through heterosexual contact. Adult HIV prevalence is 20-25 percent and has been at this level for several years. Although condom use has increased significantly since the 1980s $\mathrm{s}$, there is no evidence that HIV prevalence has started to decline. Incidence has undoubtedly declined from it peak due both to the saturation of infection in those with the

\footnotetext{
${ }^{10}$ R. S. Bernstein, D. S. Sokal, S. T. Seitz, B. Auvert, J. Stover, W. Naamara. 1998. "Simulating the Control of a Heterosexual HIV Epidemic in a Severely Affected East African City." Interfaces 28(3 MayJune): 101-26.

${ }^{11}$ E. A. Stanley, S. T. Seitz, P. O. Way, T. F. Curry, and P. D. Johnson. 1989. "The United States Interagency Working Group Approach: The IWG Model for the Heterosexual Spread of HIV and the Demographic Impact of the AIDS Epidemic." In The AIDS epidemic and its demographic consequences. Proceedings of the United Nations/World Health Organization Workshop on Modelling the Demographic Impact of the AIDS Epidemic in Pattern II Countries: Progress to Date and Policies for the Future. New York, 13-15 December.

${ }^{12}$ Steven T. Seitz and Gene E. Mueller. 1994. "Viral Load and Sexual Risk: Epidemiologic and Policy Implications for HIV/AIDS." In E.H. Kaplan and M. L. Brandeau, eds., Modeling the AIDS Epidemic: Planning, Policy, and Prediction. New York: Raven Press, Ltd..

${ }^{13}$ S. Gregson, T. Zhuwau, R. M. Anderson, S. K. Chandiwana. 1998. "Is there evidence for behaviour change in response to AIDS in rural Zimbabwe?" Social Science and Medicine 46(3):321-30.
} 
Figure 1. HIV prevalence among pregnant women in Manicaland, Zimbabwe, findings from various ante-natal

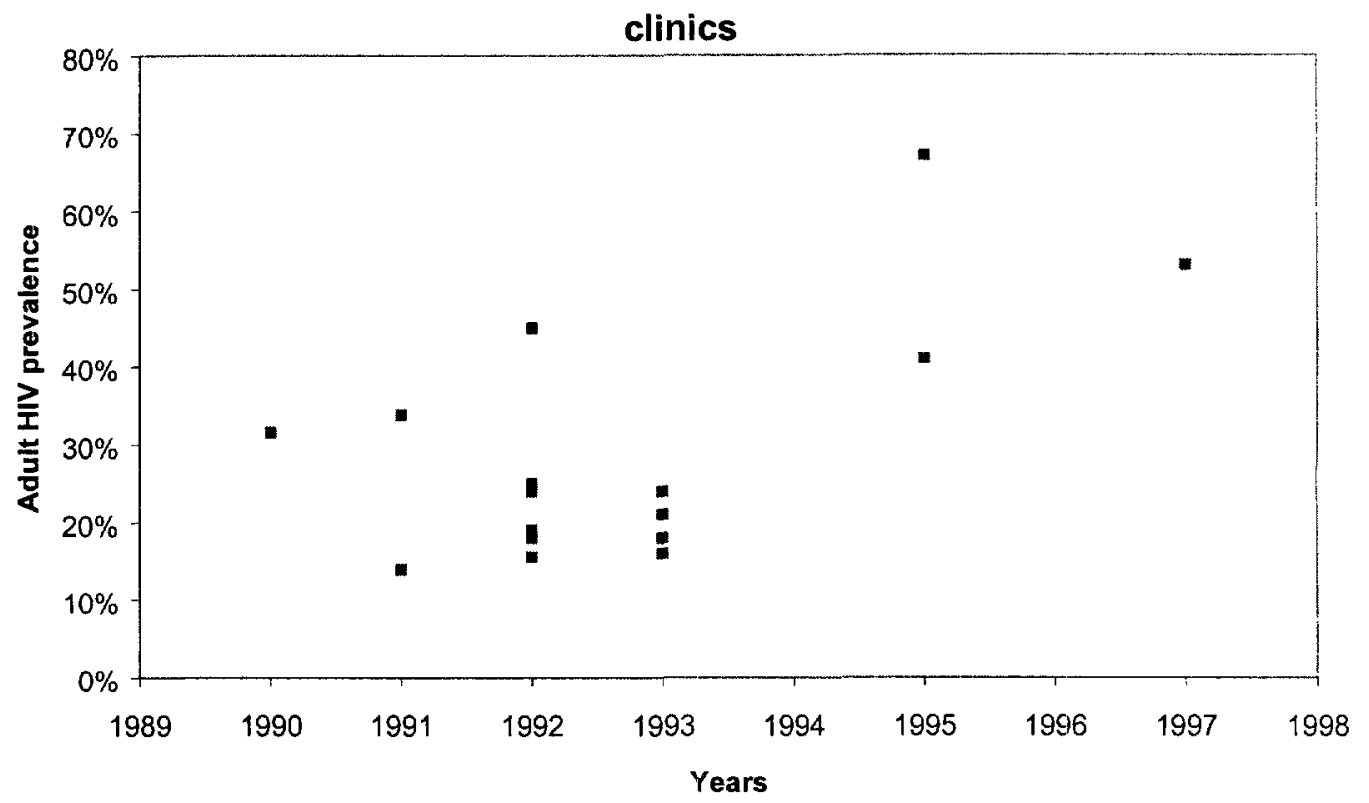

highest risk and to increased condom use, but there is no evidence yet that incidence has declined enough to cause prevalence to fall. Figure 1 shows the pattern of HIV prevalence among pregnant women attending antenatal clinics. ${ }^{14}$ Each point represents HIV infection at an individual antenatal clinic for a particular year.

\section{Kampala}

The iwgAIDS model uses Kampala, Uganda as one of its settings. The data set is based on a variety of prevalence and behavioral surveys conducted over the years. The model was first applied to Kampala in the early 1990s and has been used for several simulation exercises since then. ${ }^{15}$ The data set was updated with new information from the latest census, Demographic and Health Survey and surveillance reports.

Kampala experienced one of the earliest AIDS epidemics. HIV prevalence rose sharply during the 1980s to about 30 percent at its peak. In the 1990s prevalence declined substantially due to a combination of deaths and reduction in new infections, brought about by significant behavior change, particularly among young adults (Figure 2). ${ }^{16}$

\footnotetext{
${ }^{14}$ HIV/AIDS Surveillance Database, June 2000 Release. Washington, DC: U.S. Bureau of the Census.

${ }^{15}$ Bernstein R.S., D. S. Sokal, S.T. Seitz, B. Auvert, J. Stover, W. Naamara. 1998. "Simulating the Control of a Heterosexual HIV Epidemic in a Severely Affected East African City." Interfaces 28(3):101-26.

${ }^{16}$ HIV/AIDS Surveillance Database, June 2000 Release. Washington, DC: U.S. Bureau of the Census.
} 
Figure 2. HIV prevalence among pregnant women in Kampala

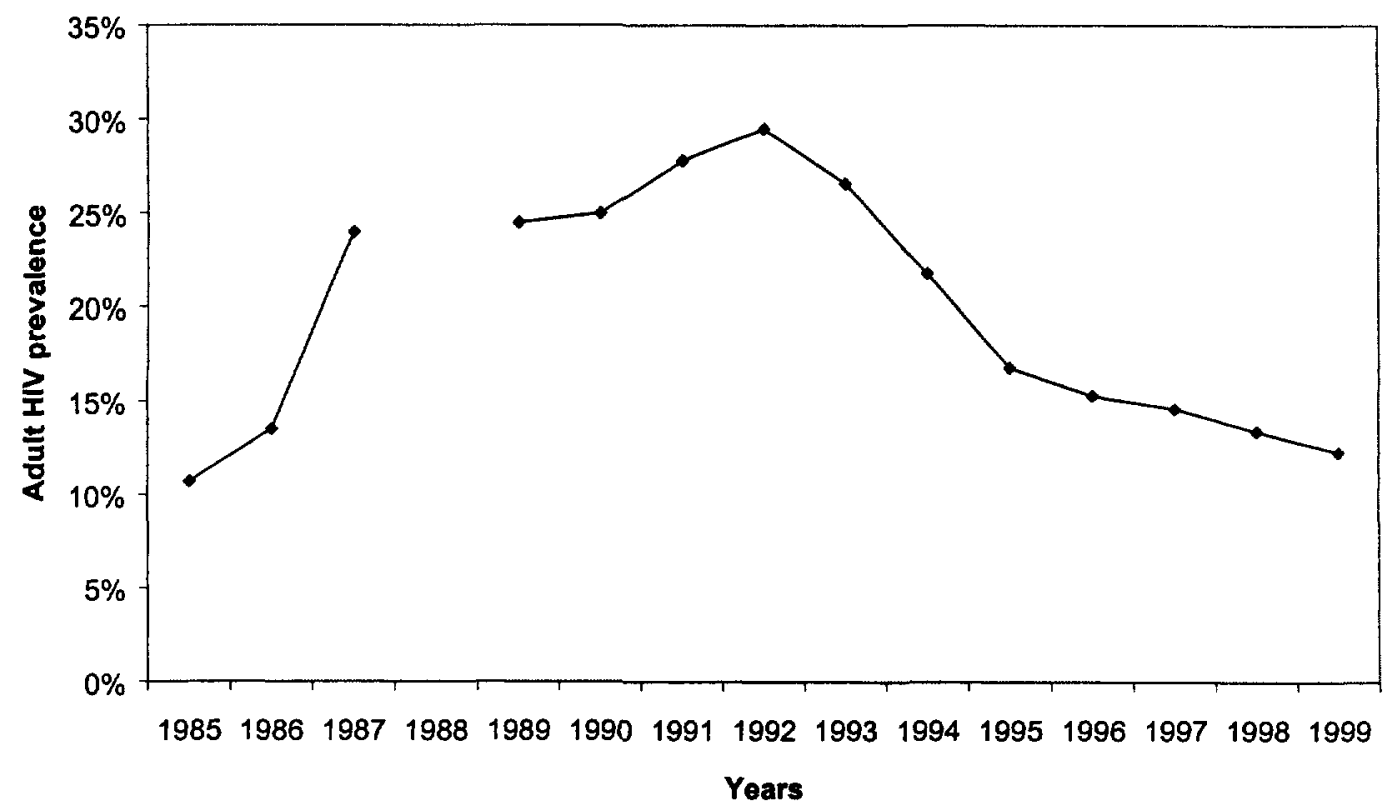

\section{Thailand}

Thailand was one of the few developing countries outside of Sub-Sarahan Africa to experience an early AIDS epidemic. HIV spreads in Thailand through a combination of heterosexual contact and injecting drug use. About one-fifth of new infections are due to injecting drug use. HIV prevalence peaked at over two percent in the early 1990s and then declined due to rising mortality and a dramatic decrease in incidence arising from the success of interventions to reduce commercial sex and encourage condom use.

Estimates of HIV prevalence among adult males and females are shown in Figure 3. ${ }^{17}$

${ }^{17}$ The Thai Working Group on HIV/AIDS Projections. 2001. Projections for HIV/AIDS in Thailand: 20002020. Division of AIDS, Ministry of Health, Bangkok.. 
Figure 3. Estimated HIV prevalence among males and females Thailand

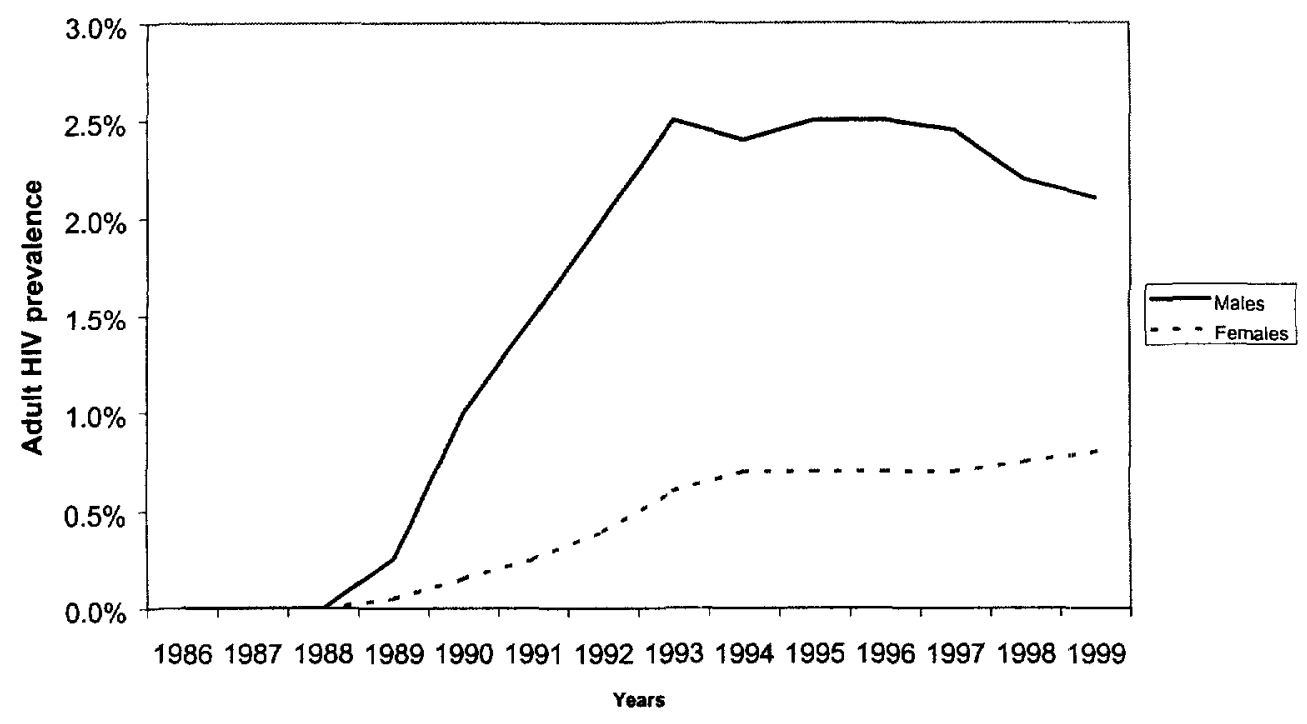

\section{Analysis}

The epidemiological impact of a vaccine will depend on where the vaccine is used (context), the characteristics of the vaccine, how it is used (strategy) and how people respond to its use.

\section{Context}

The question of where it is used is addressed by examining three different epidemics (Kampala, rural Zimbabwe, and Thailand) and by introducing the vaccine at different phases of the epidemic. These three locations illustrate different epidemic types.

Kampala is an example of a rapid heterosexual epidemic in an African city with considerable mixing among those engaging in casual sex. In the standard vaccine simulations shown below, the vaccination program starts when HIV prevalence is at its peak and HIV incidence has already declined somewhat from its peak (see box 1). Except for the early stages of the epidemic, there are more women infected than men.

Rural Zimbabwe is an example of a heterosexual epidemic in a rural area where prevalence rises to a high level (around 25 percent) and stabilizes around 20-25 percent. In the baseline and standard simulations the vaccine program is introduced during the explosive phase of the epidemic when incidence is at its peak and prevalence is rising rapidly. 


\section{Box 1. Incidence and prevalence}

Most of the results of this study are shown in terms of adult HIV incidence. Incidence is the number of new adult infections occurring each year divided by the number of adults who are not infected at the beginning of the year. Prevalence is the number of adults currently infected with HIV divided by all adults. Thus incidence is a measure of new infections while prevalence is a measure of existing infections. Both indicators are commonly calculated for the adult population 15-49. Prevalence is known more widely than incidence since it can be measured directly through surveillance surveys. Incidence is harder to measure but is a better indicator for tracking changes since it will respond more quickly than prevalence and prevalence is affected by mortality rates. The relationship between incidence and prevalence in a typical epidemic is shown in the figure below. Prevalence increases most rapidly when incidence peaks. Incidence can decline, even in the absence of behavior change, when most of those at high risk for infection are already infected. Prevalence will not decline until the number of deaths to those already infected exceeds the number of new infections.

\section{Prevalence and Incidence in a Typical AIDS Epidemic}

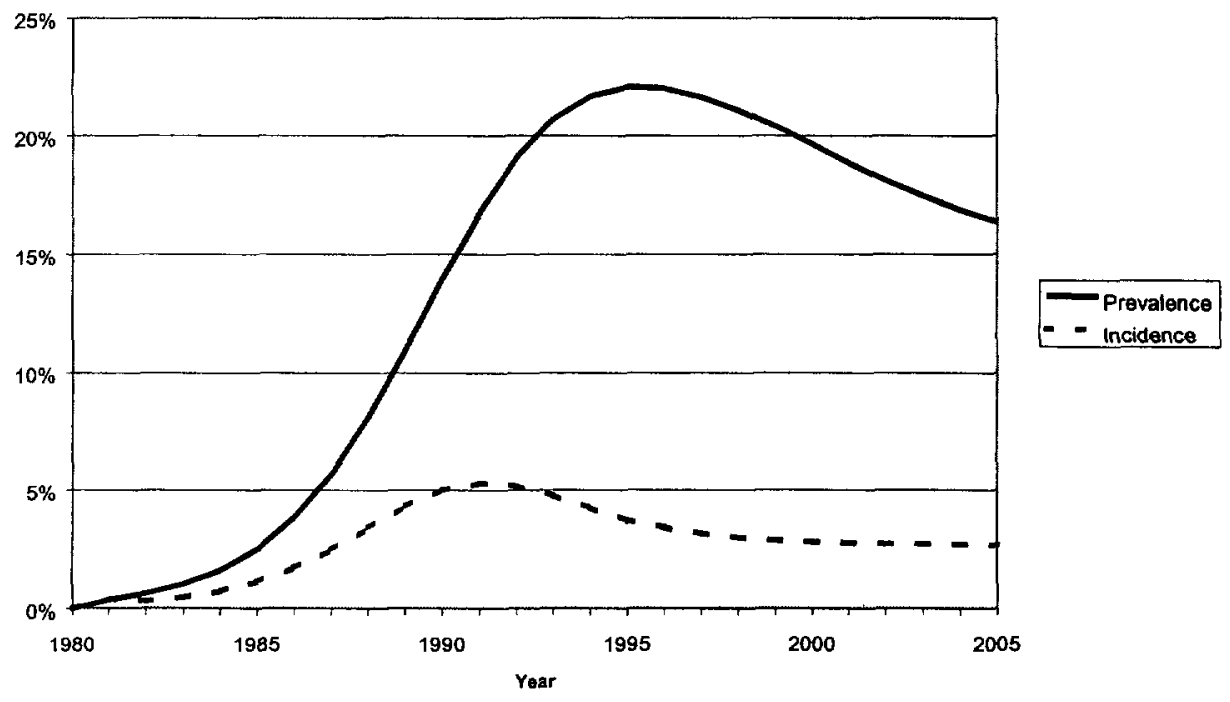

Thailand represents an Asian epidemic with a combination of heterosexual and injecting drug use transmission. Overall prevalence is much lower (about 2 percent at the peak). The epidemic is more concentrated in the highest risk groups. Throughout the epidemic there are more men infected than women. In the standard vaccine simulations the vaccination programs begins when prevalence is nearly stable and incidence is low. 


\section{Characteristics}

Various vaccine characteristics are examined in order to determine their effect on the epidemiological impact. The characteristics studied are type of protection, efficacy and duration of protection.

Type of protection refers to the action of the vaccine. A vaccine might achieve 50 percent effectiveness by completely protecting 50 percent of those vaccinated (take) or by reducing the probability of infection by 50 percent for everyone (degree). It is difficult to predict how the vaccine will operate and effectiveness studies will probably not distinguish between these two types of action. We generally assume that vaccines provide complete protection to some individuals (take) by provoking an immune system response. However, for HIV it is possible that vaccines might provide protection against initial infection. In this case, the probability of infection would be related to number of exposures and the virulence of the HIV strain, and the vaccine would provide degree type protection.

Efficacy refers to how well the vaccine protects against infection. In a take type vaccine, efficacy is the percentage of people vaccinated who are completely protected. In a degree type vaccine, efficacy is the percent reduction in probability of infection for those vaccinated. For the purposes of this study, we examined vaccines with efficacies of 50, 75 , and 95 percent.

Duration refers to the length of protection provided by the vaccine. Ideally, an HIV vaccine would provide lifetime protection. However, many vaccines provide protection for only a few years, requiring boosters to maintain protection at a high level. In this study, we examined vaccines with durations of 5 years, 10 years and lifetime. In the Imperial College model, waning vaccine protection is simulated with a mean exponential decay process, with a mean of five or ten years. In iwgAIDS duration is modeling as a median decay process. Therefore, a 5-year duration in iwgAIDS translates into somewhat longer protection than a 5 -year duration in the Imperial College model.

\section{Strategy}

Vaccines can be used in a variety of ways. Although the maximum protection would be afforded by vaccinating everyone with a lifetime vaccine that strategy may not be feasible or affordable. In this study we have assumed that vaccine coverage will reach 65 percent of the target population five years after to program starts. However, depending on the target population and duration of the protection the actual level of protection of the total adult population can be quite different from 65 percent.

If the vaccine is very expensive public subsidies for vaccination might be restricted to those at highest risk. It may be difficult to reach all adults, but easier or more costeffective to implement a program of vaccination for school children at age 15 or for women attending ante-natal clinics. In this study, we have examined the impact of vaccination programs aimed at all adults, populations at high risk of HIV infection, teenagers and adult women. 
Adult women might be the target of a vaccination campaign because they can be relatively easy to reach through ante-natal clinics, at least in Sub-Sarahan Africa where the majority of pregnant women do attend ante-natal clinics. It is also possible that a vaccine that works through mucosal immunity would only be effective in women.

For a vaccine with less than lifetime protection, it will be important to consider revaccination strategies. If the vaccine protects people during the time of highest risk (e.g., the first few years of sexual activity) then re-vaccination might not be necessary. In order to provide the highest level of protection, everyone should be re-vaccinated after a certain period of time. However, re-vaccination could be expensive. In this study, we have examined programs involving no re-vaccination and those involving re-vaccination in order to maintain coverage at 65 percent of the target group.

\section{Behavioral response}

An effective vaccination program will encourage people to get vaccinated in order to protect themselves from HIV infection. This could encourage some people to assume that once they are vaccinated they do not need to worry about unprotected sex. Some people who changed their behavior over the past few years to reduce their risk of infection, might revert to their previous behavior patterns if they think they are no longer at risk. In the worst case, some people who are not vaccinated may revert to previously risky behaviors if they believe that their risk is low because most other people have been vaccinated.

In this study we tested the effects of behavioral reversals by simulating situations in which people return to the levels of unsafe sex at the start of the epidemic. We have tested situations in which only those vaccinated revert to riskier behavior and situations in which everyone does so.

\section{Cost-effectiveness}

There is very little information on what an HIV vaccine will cost once it is ready for use. No one knows what the cost of a vaccine will be once it is ready for market. The cost will depend on the difficulty of producing the vaccine, the number of doses required to reach full effectiveness and negotiations between producers and consumers. Other researchers have assumed costs ranging from $\$ 5$ to $\$ 100$ per person vaccinated. ${ }^{18,19,20,21,22}$

\footnotetext{
${ }^{18}$ P. Cowley. 1993. "Preliminary cost-effectiveness analysis of an AIDS vaccine in Abidjan, Ivory Coast." Health Policy 24(2):145-53.

${ }^{19}$ World Health Organization and UNAIDS. 2001. "Future access to HIV vaccines. Report from a WHOUNAIDS Consultation, Geneva, 2-3 October 2000." AIDS 15(7):W27-W44.

${ }^{20} \mathrm{~V}$. Tangcharoensathien, W. Phoolcharoen, S. Pitayarangsarit, S. Kongsin, V. Kasemsup, S. Tantivess and others. 2001. "The potential demand for an AIDS vaccine in Thailand." Health Policy 57(2):111-39.

${ }^{21}$ S. Forsythe. 2001. "An Economic Evaluation of HIV/AIDS Services in Kenya: A Practical Policy Application of Contingent Valuation." The Liverpool School of Tropical Medicine.
} 
For the purpose of this analysis, we assumed a cost of $\$ 20$ per person vaccinated as the standard. Sensitivity analyses tested costs of $\$ 5$ and $\$ 100$ per person vaccinated. This includes the full cost of vaccination, including all injections, cost of delivery, wastage, booster shots, etc.

\section{Discount rate}

The costs and benefits of an AIDS vaccine will occur at different times. Vaccinations conducted today will avert infections in future years. The costs of the vaccination program can be expressed in present value by discounting future costs.

The discount rate that is used in assessing an intervention can produce critically different conclusions, particularly for an intervention such as an AIDS vaccine that would have potentially substantial long-term benefits, but might have few financial short-term consequences.

In order to put all costs into their present value, it is necessary to determine a real discount rate (nominal discount rate less inflation rate). A recent meta-analysis of 37 cost studies dealing with communicable diseases in developing countries revealed that the discount rates used varied between 2 percent and 14 percent, with a mode of 3 percent. ${ }^{23}$ The World Development Report 1993, which dealt with issues of health and development, used a discount rate of 3 percent. ${ }^{24}$ However, many World Bank projects use a discount rate of between 6 and 10 percent. ${ }^{25}$

For this study we chose a discount rate of 4 percent. Discounting is applied to both the costs of the vaccination program (dollars) and the benefits (infections averted).

\section{Cost-effectiveness}

The economic values of the vaccines studied are compared on the basis of costeffectiveness are measured by the cost per infection averted. This is calculated as the net present value of the cost of the vaccinations divided by the number of infections averted over the entire projection period. The cost of the vaccinations is simply the number of people vaccinated in each year multiplied by the cost of the vaccine per person. It should be noted that our assumption of the same vaccine price and delivery cost across all target groups is a simplification that ignores the possible cost savings through alternative targeting and delivery strategies.

\footnotetext{
${ }^{22}$ M. M. Levine, O. S. Levine.1997. "Influence of disease burden, public perception, and other factors on new vaccine development, implementation, and continued use." Lancet 350(9088):1386-92.

${ }^{23}$ D. Walker, J. A. Fox-Rushby. 2000. "Economic evaluation of communicable disease interventions in developing countries: a critical review of the published literature." Health Economics 9(8):681-98.

${ }^{24}$ World Bank. World Development Report 1993: Investing in Health. New York: Oxford University Press.

${ }^{25}$ Personal communication, Mead Over, 25 January, 2001.
} 


\section{Results}

Hundreds of simulations were made with both models to examine the impact of vaccines under different combinations of characteristics and strategies. The discussion below summarizes the key findings. Most of the illustrations in the following discussion show the impact of varying one or two factors. The results are compared against a baseline projection that does not include a vaccine.

The baseline projections for the Imperial College model (using rural Zimbabwe) and the iwgAIDS model (using Kampala and Thailand) are shown in Figures 4 and 5 . The results are shown in terms of new infections per capita starting at the beginning of the vaccination program and continuing for 15 years.

Figure 4. Baseline projection and standard vaccine for rural Zimbabwe. The standard vaccine program has 65 percent coverage of adults after five years, 50 percent efficacy, 10 years duration of protection and no behavioral reversals.

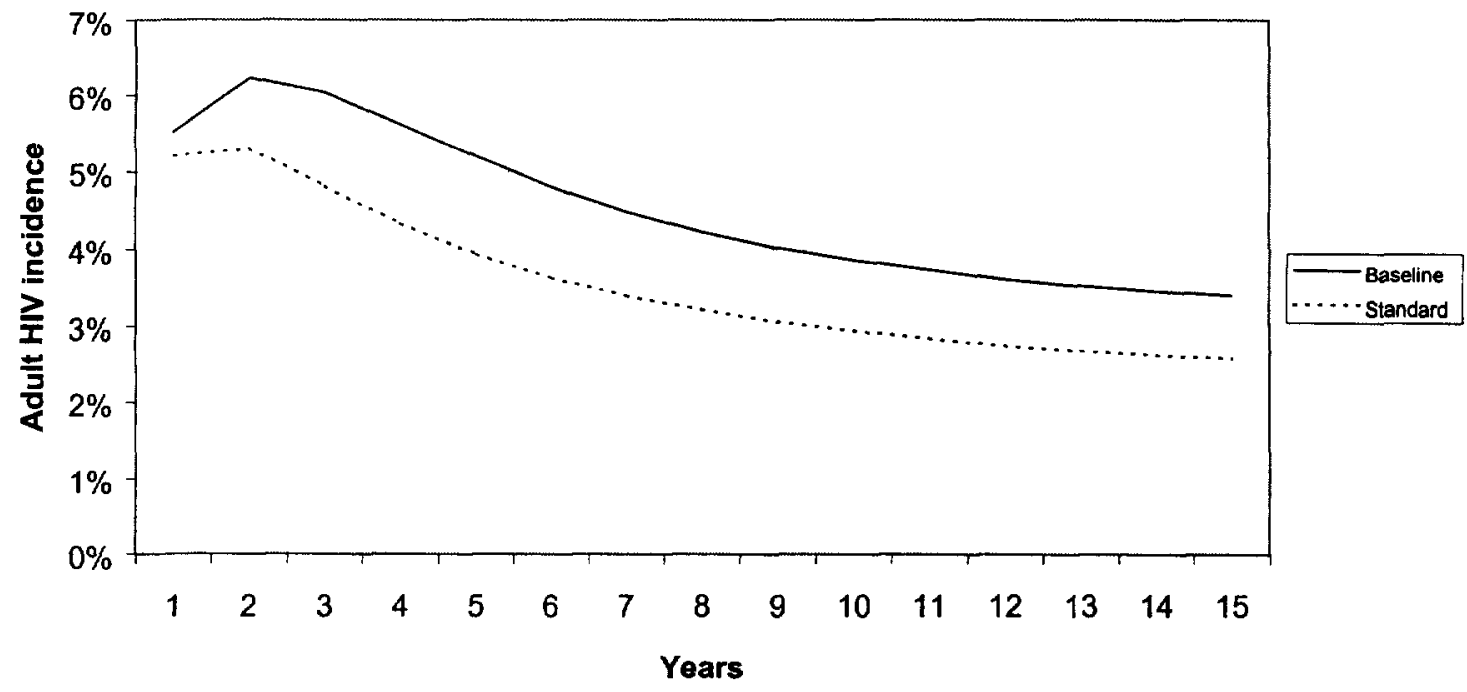


Figure 5. Baseline projection and standard vaccine for Kampala and Thailand. The standard vaccine program has 65 percent coverage of adults after five years, 50 percent efficacy, 10 years duration of protection and no behavioral reversals.
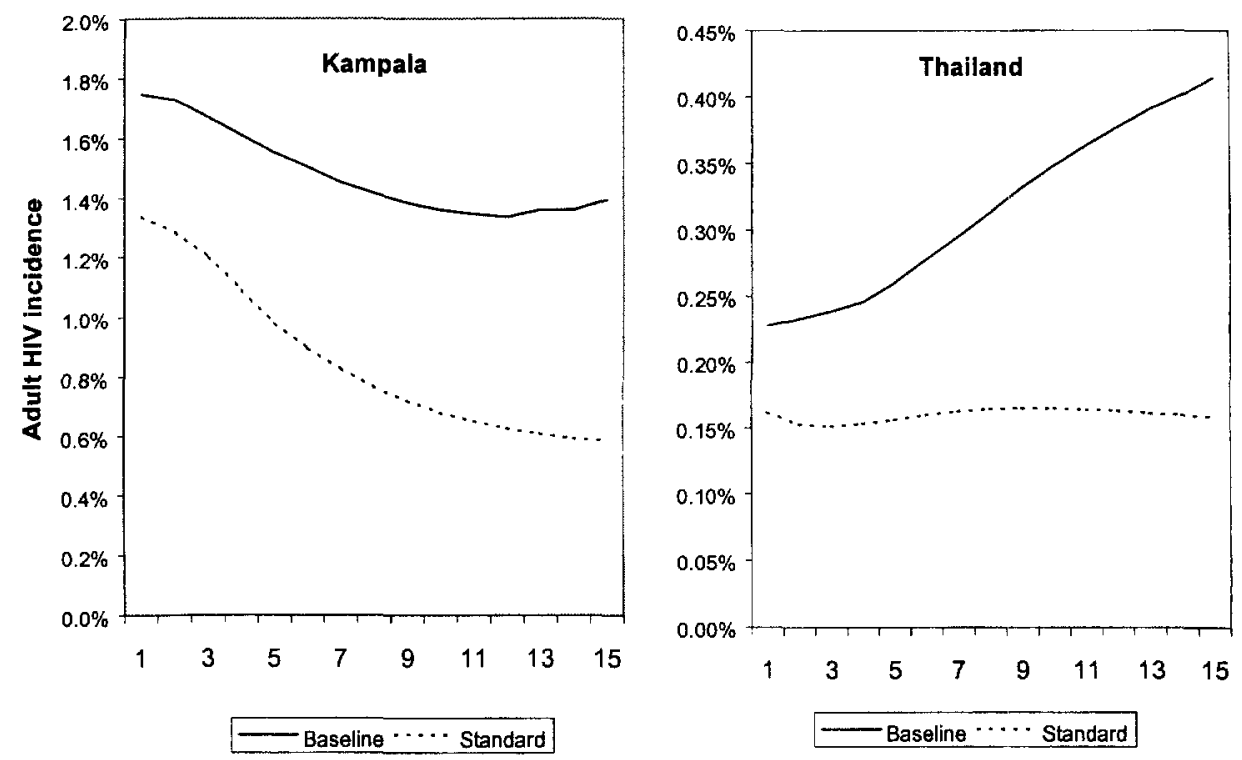

\section{Standard vaccine}

We have defined a "standard" vaccine to use for comparing the impacts of variations in vaccine characteristics and programs. The standard vaccine has degree type protection, 50 percent efficacy and 10 years duration of protection. Coverage grows to reach 65 percent of all adults 15-49 five years after the start of the program. ${ }^{26}$ After year five new vaccinations occur at a rate to cover 65 percent of the population growth. These vaccinations are given to new entrants into the adult population and older adults who were not vaccinated earlier or whose protection from an earlier vaccination has waned. Thus, 65 percent coverage means that roughly 65 percent of adults have ever been vaccinated, even though for some, the vaccination may no longer be providing protection.

The standard program assumes no behavior change as a result of vaccination.

As Figures 4 and 5 show the impact of this standard vaccine is large. It reduces adult HIV incidence by 60 percent in Thailand and Kampala and by about 25 percent in Zimbabwe. Incidence is not reduced to zero however. Since coverage is only 65 percent, efficacy is

\footnotetext{
${ }^{26}$ Note that this coverage figure applies only to susceptible adults. Some infected people might also be vaccinated. If so, the number of people vaccinated and the costs of the programs would be higher than shown here, but the number of infections averted would be the same. If adults are vaccinated regardless of their HIV status, then the number of people vaccinated would be somewhat higher than shown here depending on HIV prevalence. It would be about 1 percent higher for Thailand and about 10-15 percent higher for Kampala and Zimbabwe.
} 
only 50 percent and the duration of effectiveness is only ten years, many people are left unprotected by the vaccine.

The cost per infection averted is relatively good for the standard program particularly at the low (\$5/person) and medium (\$20/person) cost estimates, as shown in Table 1.

Table 1. Cost-effectiveness of the standard vaccine program (US Dollars per infection averted)

\begin{tabular}{lccc}
\hline & Low & Medium & High \\
Setting & $\$ 5 /$ person & $\$ 20 /$ person & $\$ 100 /$ person \\
\hline Rural Zimbabwe & $\$ 70$ & $\$ 290$ & $\$ 1,430$ \\
Kampala & $\$ 70$ & $\$ 280$ & $\$ 1,400$ \\
Thailand & $\$ 350$ & $\$ 1,410$ & $\$ 7,050$ \\
\hline
\end{tabular}

\section{Characteristics}

\section{Type of protection - take versus degree}

In certain settings the type of protection, take or degree, could make a large difference to the impact. Since we will probably not know at first which type of protection is provided by a particular vaccine, it is useful to know whether the type of protection affects the usefulness of the vaccine. For example, in a population at very high risk, such as commercial sex workers, a vaccine that reduced the probability of infection for each individual by 50 percent would not have much impact on HIV prevalence since most would still become infected. However, a vaccine that fully protected half of those vaccinated would reduce HIV prevalence by at least that much.

Type of protection is also very important in cases where behavioral reversal takes place. With a take type vaccine, behavior reversal among those effectively protected by the vaccine will not matter at all. With a degree type vaccine, behavior reversal could offset much of the protective effect of the vaccine.

In larger populations, where average risk is much lower, the difference in impact between the two types of vaccines becomes less. In this case, a reduction in the probability of infection can protect some people from ever becoming infected, just as if they were fully protected. Figure 6 compares the baseline scenario with a vaccine with take or degree protection at 50 percent efficacy. After five years, when vaccine coverage reaches 65 percent, HIV incidence is about 32 percent $(0.50 \times 0.65)$ lower than it would be without the vaccine with either take or degree action. In the longer run take type protection will produce somewhat better results since it will work equally well among high-risk and lowrisk populations while degree vaccines will only achieve the full 50 percent effectiveness among low-risk populations. Take type protection is clearly better, but a vaccine with degree type protection would still be very useful. 
Figure 6. The effects of type of protection on HIV incidence in rural Zimbabwe. Take protection completely protects some individuals, degree protection reduces the probability of infection for everyone receiving the vaccine

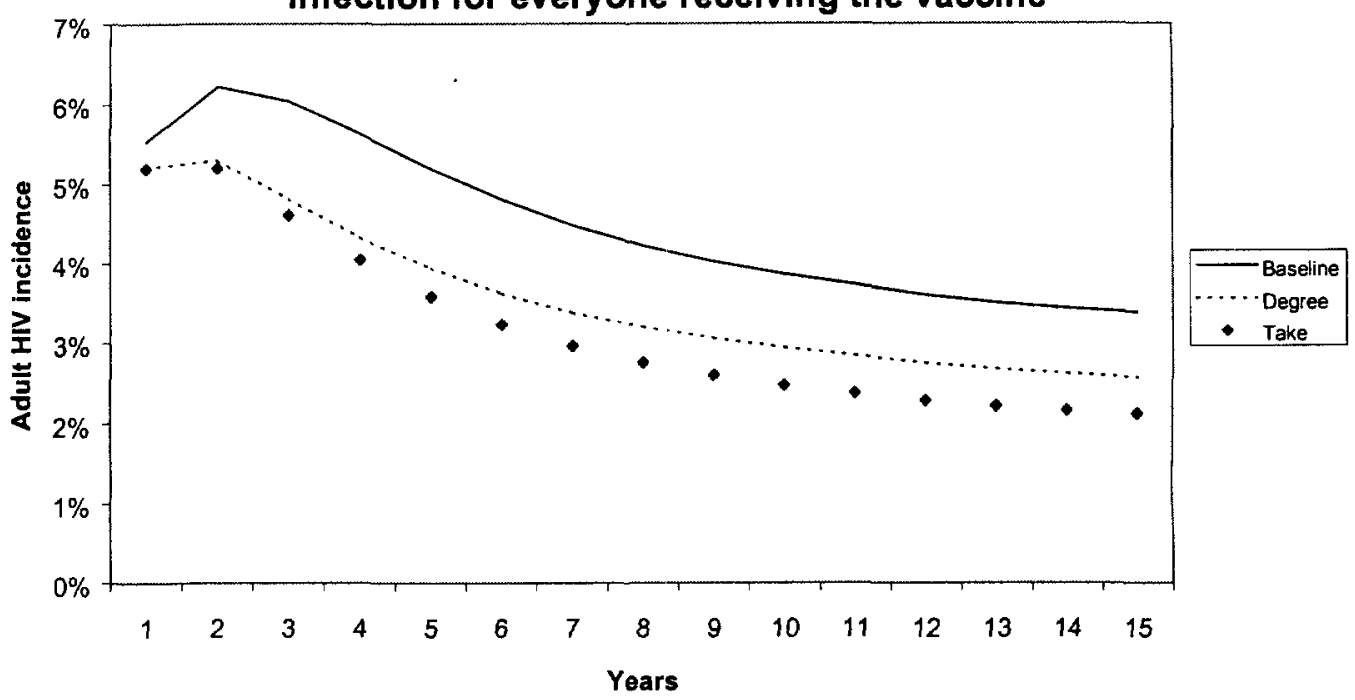

\section{Efficacy}

Figures 7 and 8 show the impacts of vaccines with different levels of efficacy. At 95 percent efficacy, the vaccination program can nearly extinguish the epidemic. Clearly better efficacy produces greater impact. However, even 50 percent efficacy provides a large benefit when 65 percent of adults are vaccinated. In the settings we investigated there is no evidence of a threshold value below which a vaccine would be nearly useless. In these simulations the impact increases regularly with effectiveness. It is possible that in very low prevalence epidemics there might exist a threshold above which the epidemic would be extinguished. Any threshold value would be sensitive to the level of coverage.

In the simulations shown in Figure 7 and 8, the efficacy of the vaccine does not affect the costs of the program since the same numbers of people are vaccinated in each simulation. However, more infections are averted with greater efficacy so the benefits are higher as shown in Table 2.

Table 2. The effect of efficacy on cost per infection averted, assuming a cost of $\$ 20$ per person vaccinated (US Dollars per infection averted)

\begin{tabular}{lccc}
\hline Efficacy & Rural Zimbabwe & Kampala & Thailand \\
\hline 50 percent & $\$ 290$ & $\$ 280$ & $\$ 1,410$ \\
75 percent & $\$ 160$ & $\$ 180$ & $\$ 960$ \\
95 percent & $\$ 110$ & $\$ 150$ & $\$ 780$ \\
\hline
\end{tabular}


Figure 7. Effects of vaccine efficacy on HIV Incidence in rural ZImbabwe

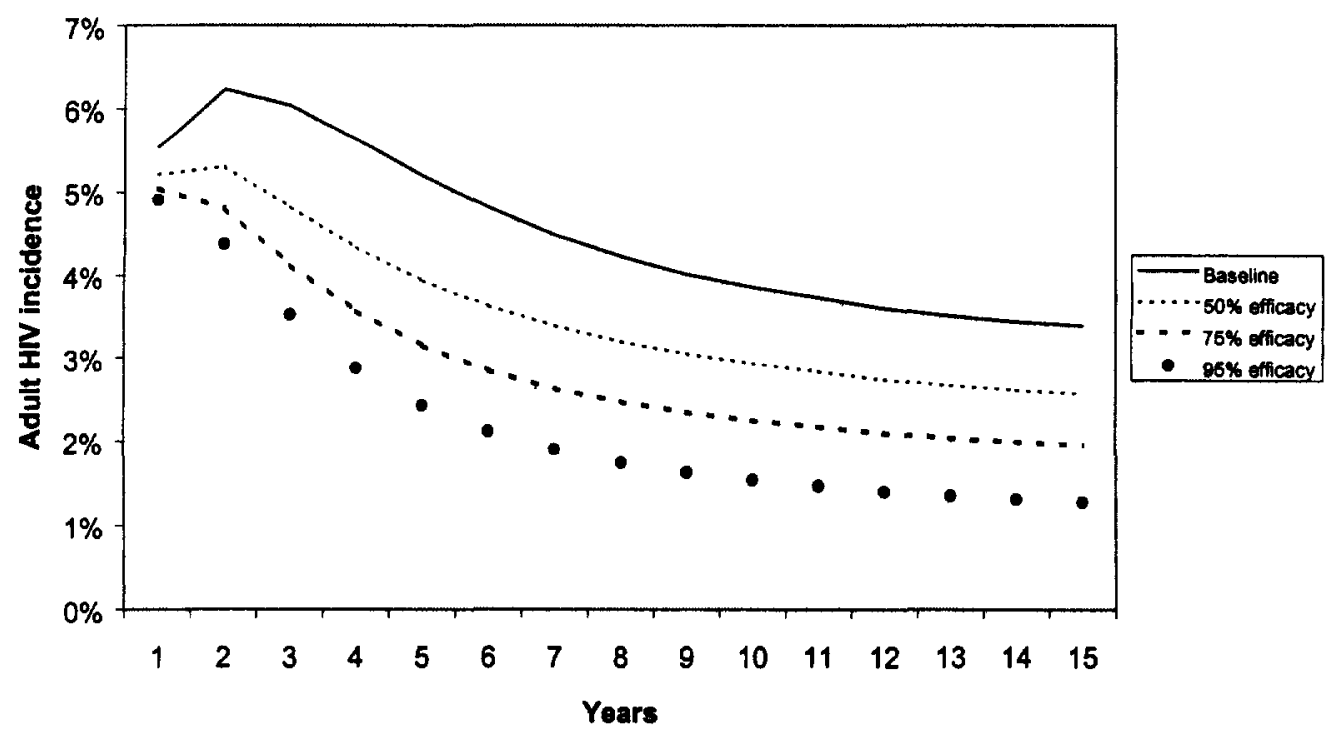

Figure 8. Effects of vaccine efficacy on HIV incldence in Kampala and Thailand
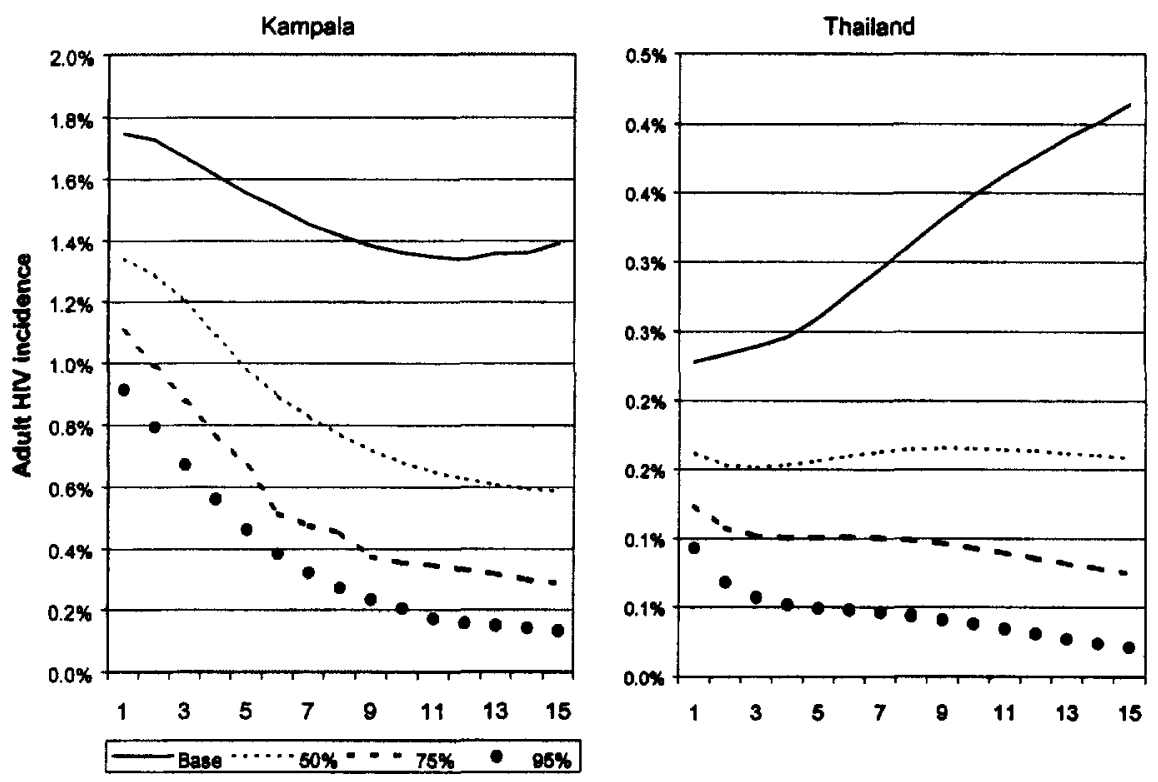


\section{Duration}

We have examined the effect of duration of protection by simulating vaccines with durations of 5 years, 10 years and lifetime. The impact of these different durations depends on how the vaccine is implemented and the context in which it is implemented. At one extreme imagine a vaccine that is given only to 15 year olds and protects for only five years. It would provide protection during the high-risk adolescent phase, but no protection after that. A vaccine with lifetime protection given to 15 year olds would produce a much larger impact. However, a strategy of re-vaccinating people every five years would produce almost as much impact as a lifetime vaccine. Its impact would still be somewhat less because of the difficulty of re-vaccinating everyone before protection wanes.

Figures 9 and 10 illustrate the effect of duration. In Figure 9, the short duration vaccine as implemented in the Imperial College model has an important impact, but its effect is only half that of a lifetime vaccine. In this model, when vaccine protection wanes people are eligible for re-vaccination but only after a two year delay. Thus a vaccine with short duration exposes people to much more risk during their lifetime than one with longer duration.

In Figure 10, the iwgAIDS model applied to Kampala and Thailand shows very little effect of changes in duration. In this model, risk is high during adolescence and then drops substantially for most people once a stable union is formed. Even a short duration vaccine can protect people during the period of highest risk. As a result the difference between long and short duration vaccines is not as great as with the Imperial College model. However the effect of duration becomes more important if vaccinations are targeted just at teenagers (not shown). In this case, the vaccine still provides protection

Figure 9. Effects of vaccine duration on HIV incidence in rural Zimbabwe

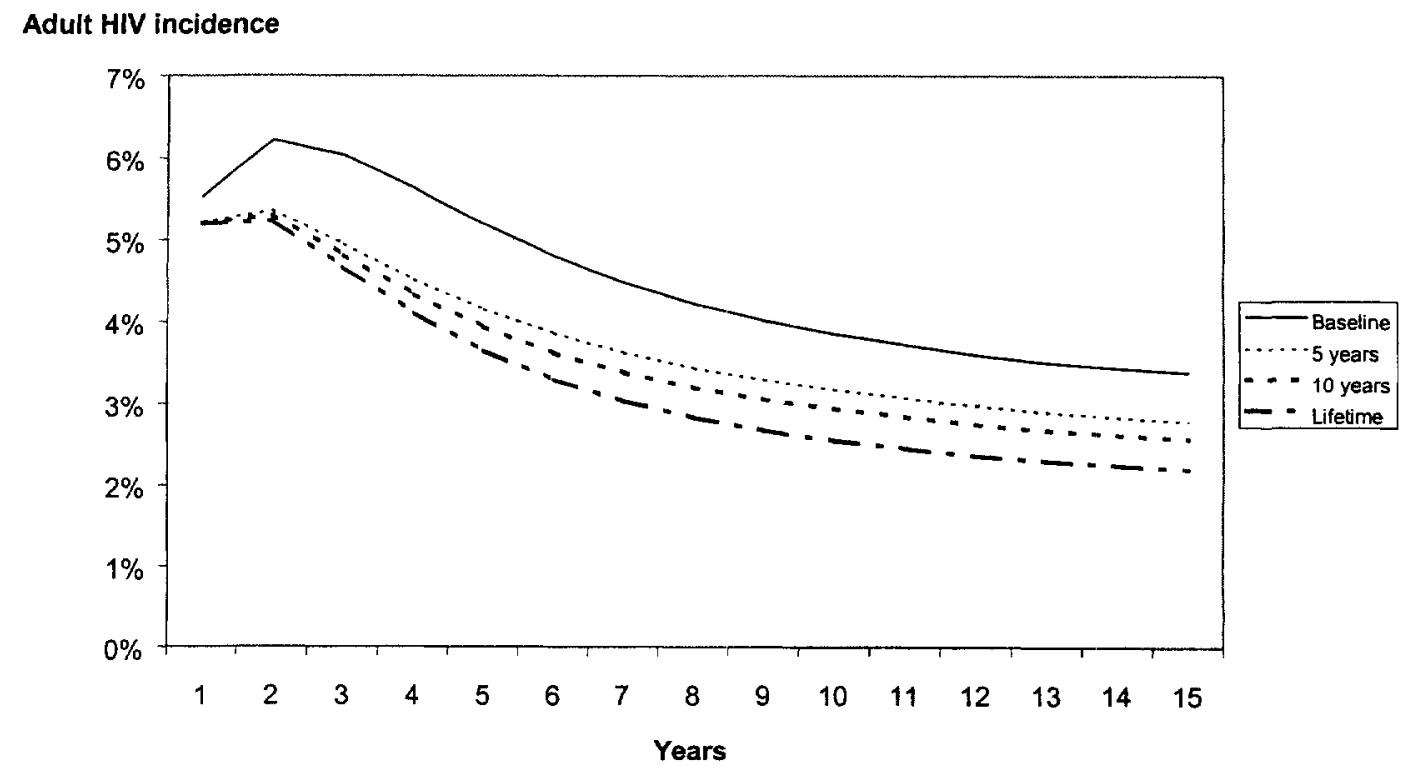


Figure 10. Effects of vaccine duration on HIV incidence in Kampala and Thailand
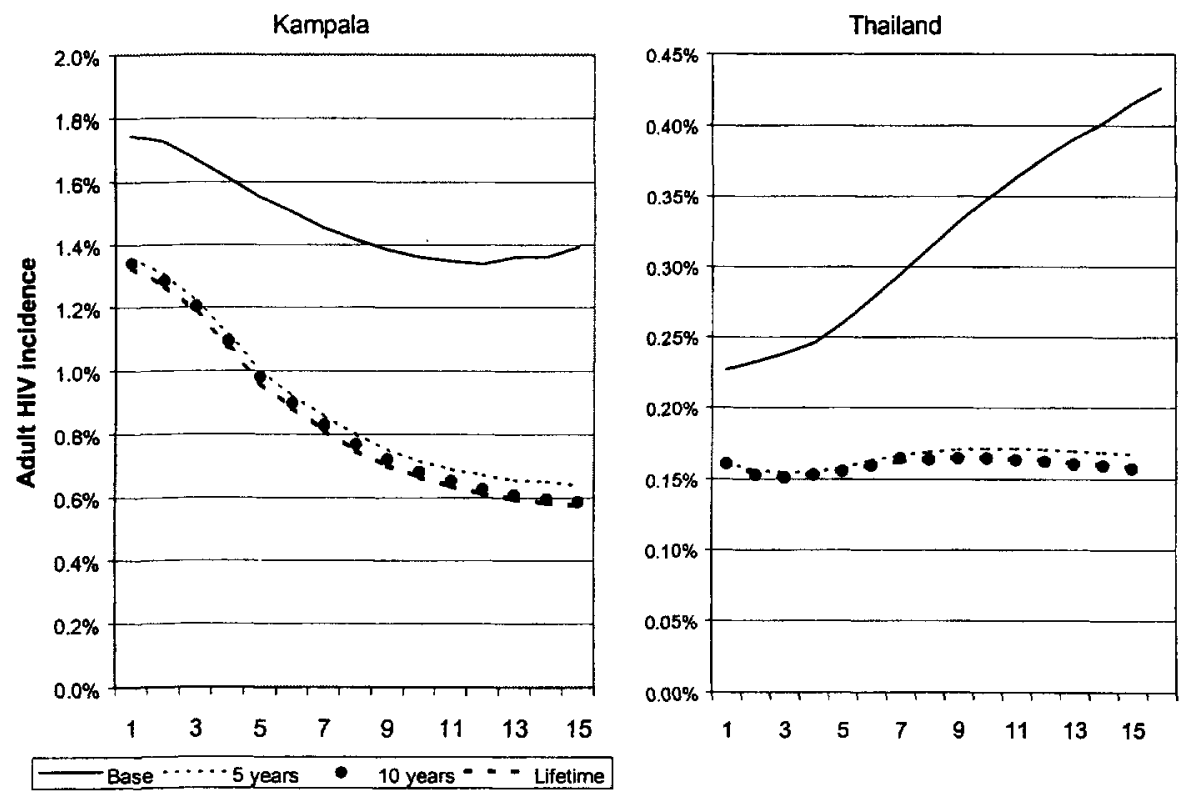

during the ages of highest risk, but since the vaccine does not reduce prevalence among older adults, the teenagers quickly become exposed to a significant risk of infection once the vaccine protection wanes.

The cost per infection averted is affected by the duration of protection. If there is no massive revaccination program, then the costs would be similar across all three scenarios of duration but the longer duration vaccines would avert more infections. These results are shown in Table 3.

Table 3. Cost-effectiveness versus duration of protection, assumes a cost of $\$ 20$ per person vaccinated (US Dollars per infection averted)

\begin{tabular}{lccc}
\hline Duration of protection & Rural Zimbabwe & Kampala & Thailand \\
\hline 5 years & $\$ 390$ & $\$ 310$ & $\$ 1470$ \\
10 years & $\$ 290$ & $\$ 280$ & $\$ 1410$ \\
Lifetime & $\$ 190$ & $\$ 260$ & $\$ 1410$ \\
\hline
\end{tabular}

Clearly a vaccine with lifetime protection is preferred. However, if only a short-term duration vaccine is available it will be necessary to devise appropriate re-vaccination strategies to account for the short duration. This will raise the costs of the program for short duration vaccines relative to longer duration vaccines. The size of this effect depends on the pattern of risk by age. If most risk is concentrated in the first few years of sexual activity before marriage, then a short duration vaccine will be almost as effective as one with lifetime protection. In populations where a significant proportion engage in risky behavior throughout their lifetime, then a short duration vaccine will be much less effective than one with lifetime protection. 


\section{Strategy}

The standard simulation assumes that the vaccination program reaches 65 percent coverage for adults within five years. Many variations on the standard approach are possible.

\section{Timing}

The HIV/AIDS epidemic is at different stages in different countries. Our standard simulation for rural Zimbabwe assumes that the vaccination program starts during the explosive phase of the epidemic, when prevalence is rising rapidly. If the start of the program is delayed until year 15 , when prevalence has just about peaked, eventual impact on incidence will be the same as for the earlier start (Figure 11). In general, the effects of the vaccine are similar whenever the vaccination program is started. Clearly the earlier the vaccination program can be started, the better. However, a vaccination program will still show significant benefits even if the epidemic is already well advanced once a vaccine becomes available.

\section{Targeting teenagers}

The effect of vaccinating just young adults can be seen in Figures 12 and 13. The impact of vaccinating just teenagers is about half as large as vaccinating all adults. Although the total effect is less, the cost-effectiveness of this strategy is better in Thailand and Zimbabwe since many fewer people would be vaccinated (Table 4). The opposite is true in the Kampala simulation. There are two reasons for this difference. First, the simulation for Kampala assumed much higher fertility than the simulations for rural Zimbabwe or Thailand, so there are many more teenagers needing vaccination. Second, the simulation with the iwgAIDS model does not vaccinate everyone at the exact age 15, but rather a

\section{Figure 11. Effects of phase of the epidemic at the start of the vaccination program on HIV incidence in rural \\ Zimbabwe}

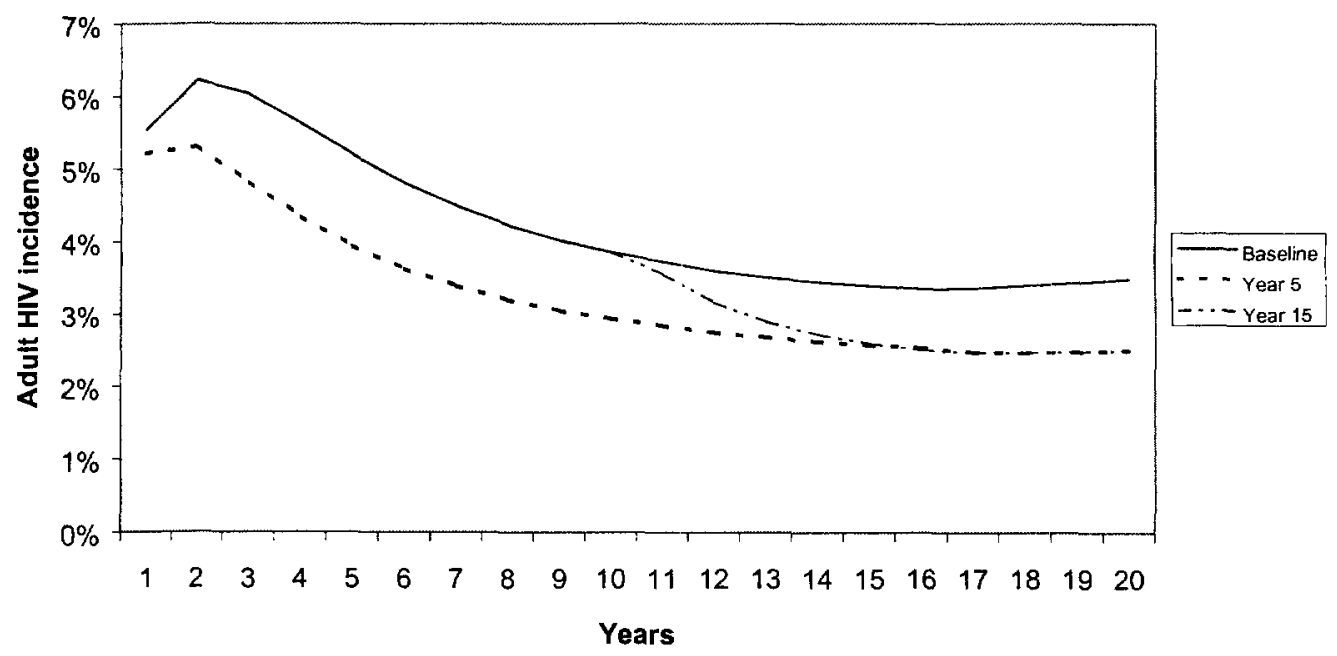


distribution around age 15 , from 13 to 18 . Once the initial program vaccinates most of the 13-18 year olds, new vaccinations will take place mainly among the youngest age groups. If the median duration of protection is only 10 years, much of this protection will wane while these young people are still in their late teens and early $20 \mathrm{~s}$, when risk may still be high.

This illustrates a difficult implementation issue. It would be ideal to vaccinate teenagers just before they become sexually active, especially with a short duration vaccine. But vaccination would probably happen by school class. So everyone would be vaccinated at the same age. That age would have to be low enough to protect most people before they become sexually active. But at a low age, vaccine protection would already be waning by the time some teenagers become sexually active. Thus, depending on the risk profile, targeting teenagers might be more or less cost-effective than targeting adults in general, especially if the duration of protection is short.

In the case of a vaccine of lifetime duration, then the strategy of focusing on teenagers would eventually produce the same effect as vaccinating all adults, but it would take 25 years to achieve this result.

Figure 12. Effects on adult HIV prevalence of targeting vaccination to 15-year olds or all adults in rural Zimbabwe

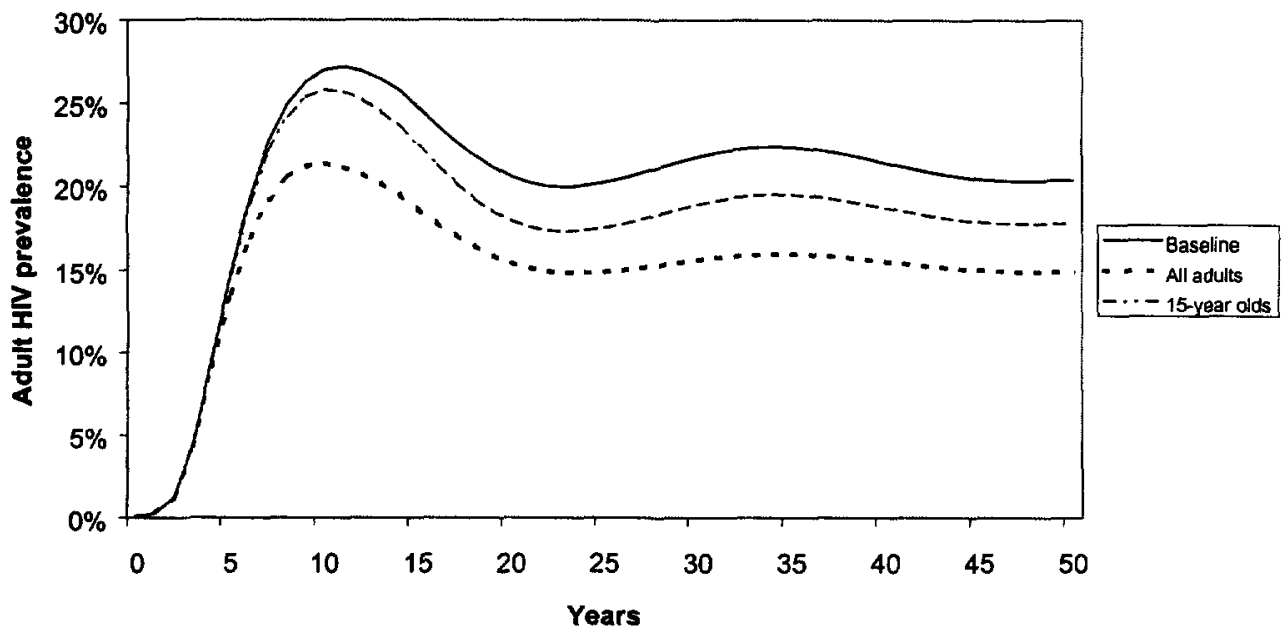


Figure 13. Effects on HIV incidence of targeting vaccination to teenagers or all adults in Kampala and Thailand
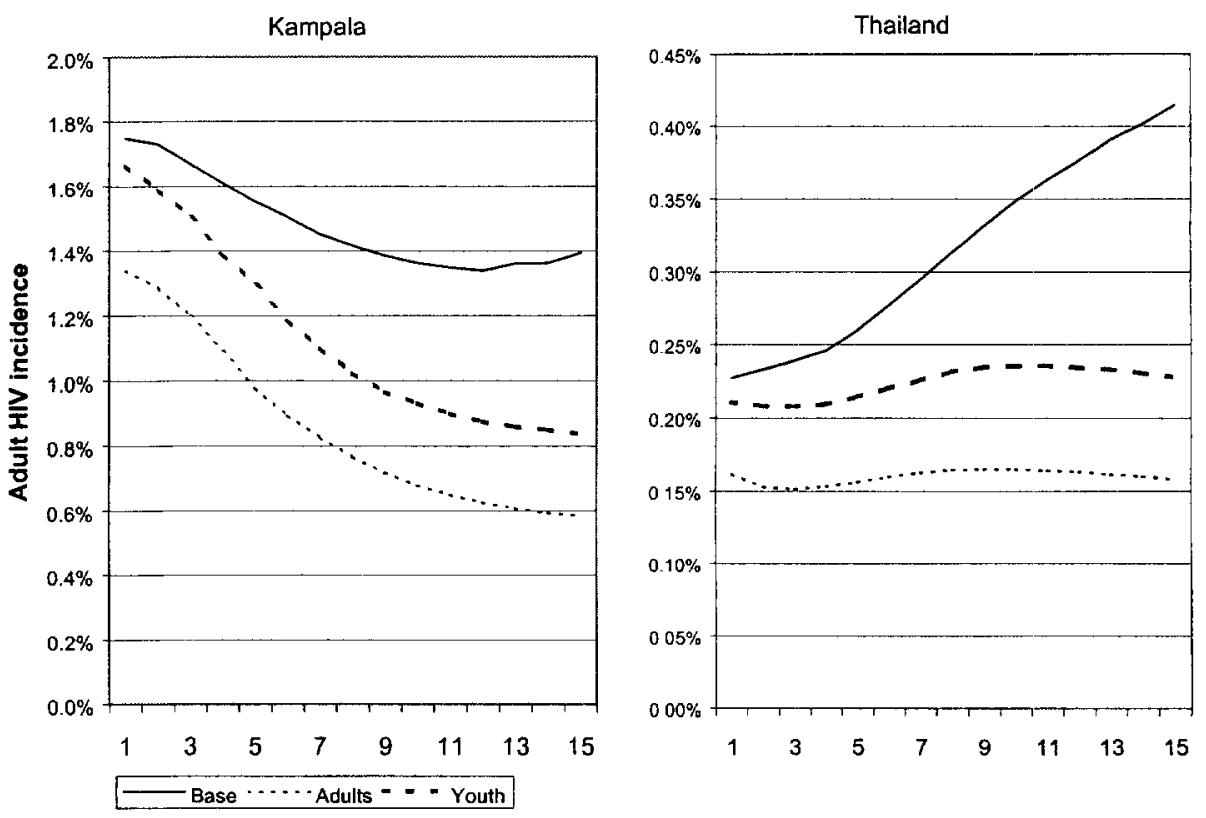

\section{Targeting high-risk groups}

The effect of targeting a vaccination program just to people with the highest risk behaviors can be seen in Figures 14 and 15. In the rural Zimbabwe simulations shown in Figure 14 only the very highest risk individuals, amounting to about 2 percent of the population, are vaccinated. In this case the effect is not large, since with a vaccine with degree protection, many of those vaccinated will still become infected. In the Kampala and Thailand simulations shown in Figure 15, a broader definition of high risk is used encompassing approximately 20 percent of adults, so that a larger proportion of the population is vaccinated. The impact is larger in this case, with about half the effect of vaccinating all adults..

The number of vaccinations is much less in this strategy so the cost of the program is reduced if we assume that the cost per person is the same in either case. If it actually costs more to reach high-risk populations, then the difference in cost between targeting all adults and just high-risk populations would be less. In the case of similar cost per person reached, the cost per infection averted can be higher when the highest-risk populations are targeted (Table 4 results for rural Zimbabwe). This result differs from findings for other prevention interventions, which show that targeting high-risk populations is always more cost-effective than general population programs although the total effect is less. (The reason for the difference is that other prevention programs, such as condoms, protect both the sex worker and the client, while a vaccine would protect only the sex worker. If the sex worker is already infected or becomes infected, there would be no protection for the client.) However, this conclusion only holds for vaccines 
of degree type protection. With take type protection, targeting high-risk populations is more cost-effective than the standard program.

We would not expect any program to vaccinate just high-risk individuals. However, public subsidies might be targeted to increase the vaccination rate among high-risk populations, particularly if vaccines are very expensive. These simulations show the extreme case where only high-risk populations are vaccinated.

Table 4. The effects of targeting on vaccine cost-effectiveness, assuming a vaccine that costs $\$ 20$ per person vaccinated (US Dollars per infection averted)

\begin{tabular}{lcccc}
\hline & $\begin{array}{c}\text { Rural Zimbabwe } \\
\text { (Degree } \\
\text { protection) }\end{array}$ & $\begin{array}{c}\text { Rural Zimbabwe } \\
\text { (Take } \\
\text { protection) }\end{array}$ & $\begin{array}{c}\text { Kampala } \\
\text { (Degree } \\
\text { protection) }\end{array}$ & $\begin{array}{c}\text { Thailand } \\
\text { (Degree } \\
\text { protection) }\end{array}$ \\
\hline $\begin{array}{l}\text { Adults } \\
\text { Teenagers }\end{array}$ & $\$ 290$ & $\$ 210$ & $\$ 280$ & $\$ 1,410$ \\
High risk & $\$ 90$ & $\$ 70$ & $\$ 460$ & $\$ 1,250$ \\
$\begin{array}{l}\text { Reproductive } \\
\text { age women }\end{array}$ & $\$ 190$ & $\$ 80$ & $\$ 190$ & $\$ 1,090$ \\
\hline
\end{tabular}

Figure 14. Effects of targeting highest risk populations on HIV incidence in rural Zimbabwe

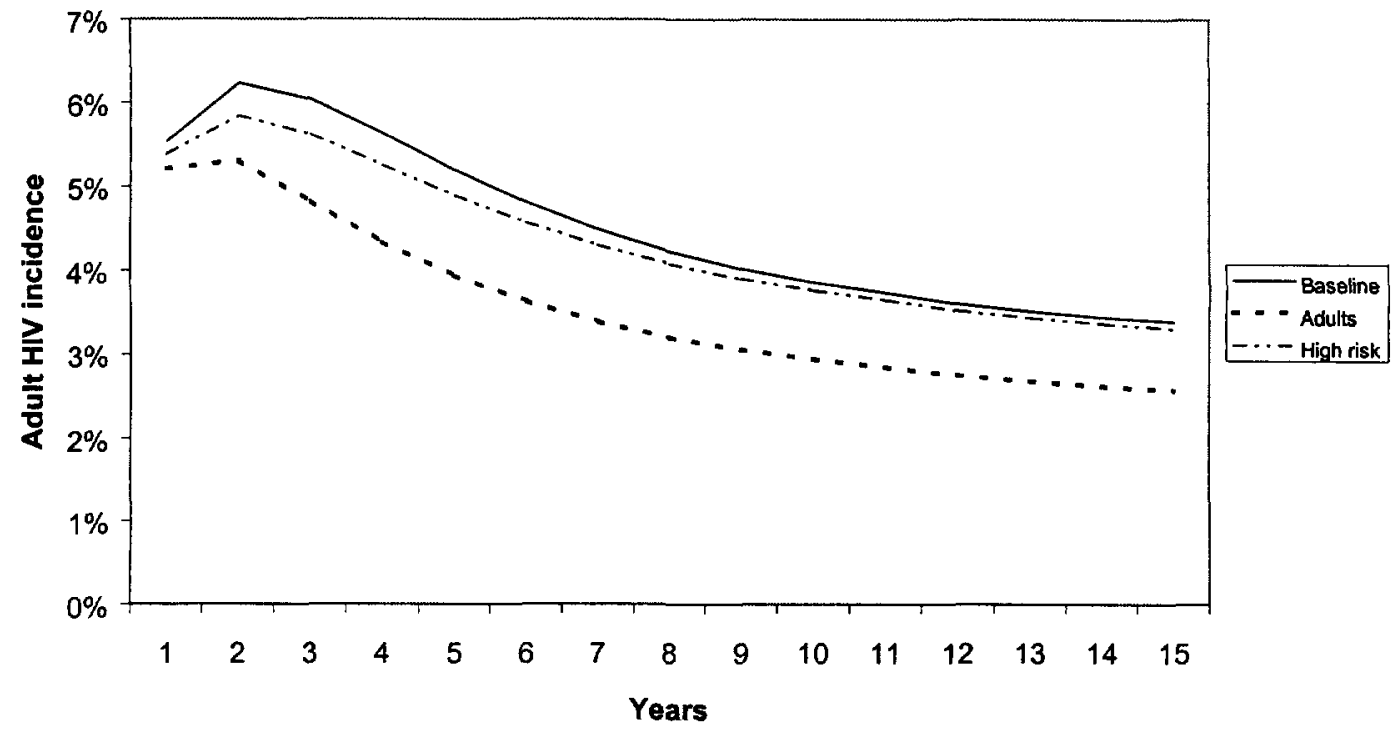


Figure 15. Effects on HIV incidence of targeting vaccination to high risk populations in Kampala and Thailand
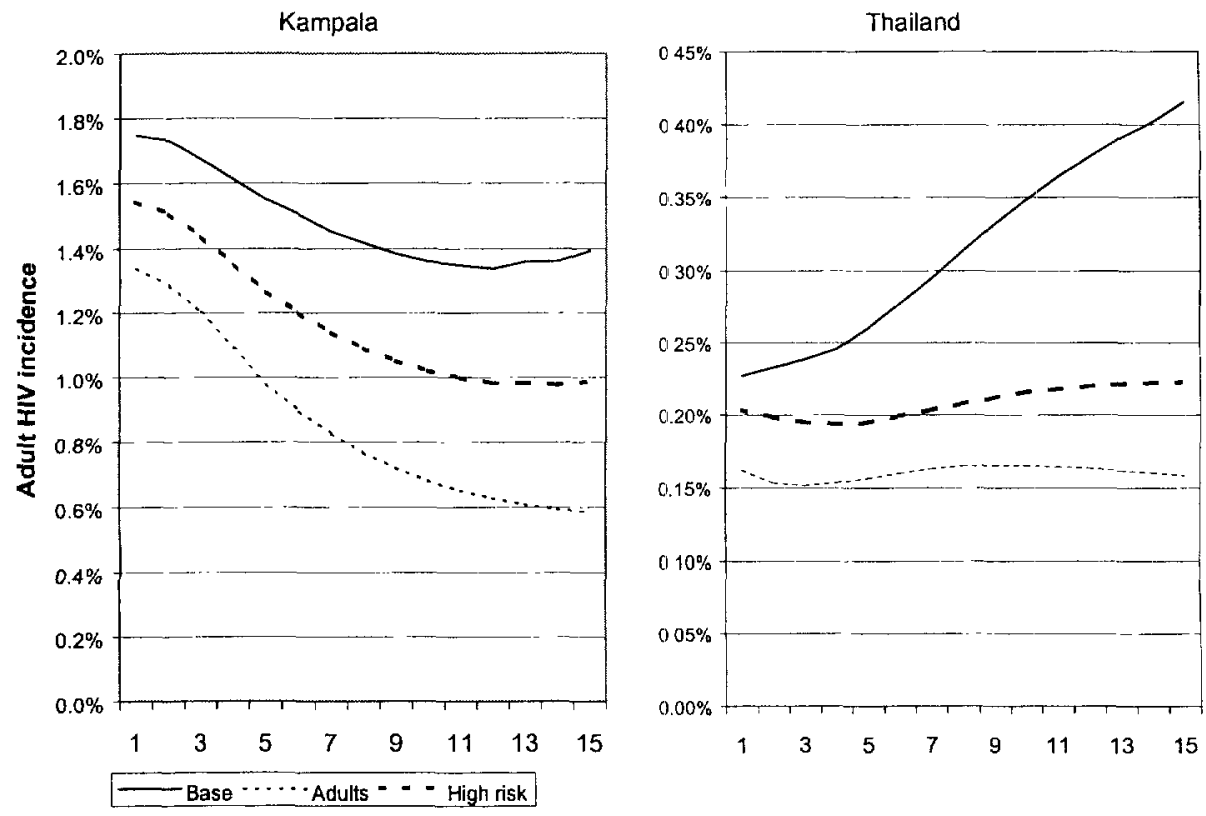

\section{Targeting women}

Figure 16 shows the impact of a program that targets women. ${ }^{27}$ Even though roughly the same number of women are being vaccinated under this program as with the standard intervention, the effect is only half as great, since women are not also protected by a reduced risk among men. In this case, no men are vaccinated but the number of male HIV infections is reduced because of reduced transmission from women.

In the Kampala simulation the cost-effectiveness of targeting women is much better than in Thailand. This is due to the high fertility rate in Kampala. Each infection averted in a reproductive age woman can potentially avert infections in newborns as well. When fertility is quite high, each infection averted in a woman might also avert one or more child infections, greatly increasing the cost-effectiveness of the program.

\footnotetext{
${ }^{27}$ This might be achieved by providing vaccinations to women attending antenatal clinics, but the simulations shows here assume that vaccination is provided to all women regardless of pregnancy status or attendance at antenatal clinics.
} 
Figure 16. Effects on HIV incidence of targeting vaccination to reproductive age women in Kampala and Thailand
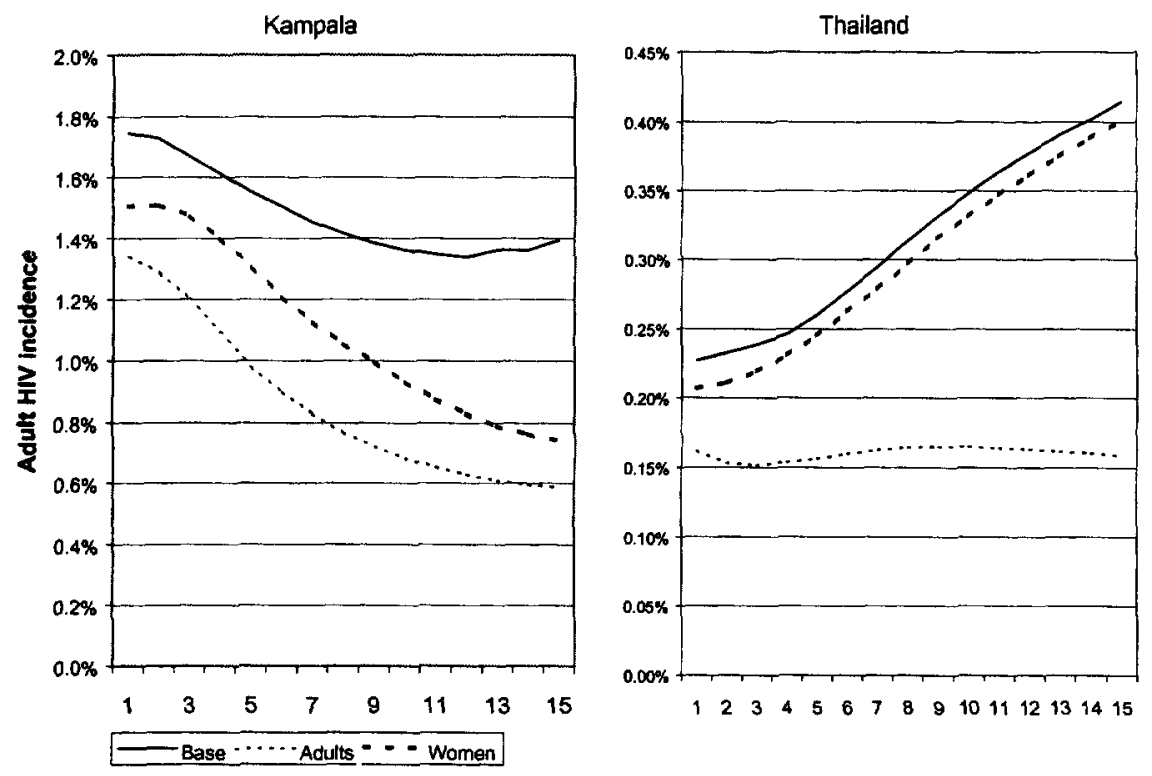

Although there would be some value to implementing a vaccine that only works in women, the strategy of vaccinating at antenatal clinics might not be the best approach. Although all women attending antenatal clinics are sexually active and, therefore, at some risk of infection, they are not typically the highest risk women. As a result, coverage rates would have to be very high to have a significant effect. With a vaccine of 95 percent efficacy, instead of the 50 percent in the standard simulation, the impact would be larger, about double the impact of the standard simulation (not shown). On the other hand, the majority of women at ante-natal clinics may be low-risk women who would be less likely to adopt riskier behaviors in response to being vaccinated. So the impact of the vaccine would not be eroded by behavioral change as might happen with high-risk populations.

\section{Coverage strategies}

The effects of various approaches to providing vaccination coverage are shown in Figure 17. The cohort vaccination program only vaccinates 15 year olds. With the short duration of protection, the benefits of this program are small. In the catch-up and cohort campaign 21 percent of all susceptible adults are vaccinated in each of the first five years, to bring coverage to 65 percent, and then 65 percent of 15 year olds are vaccinated each year. Since the duration of protection is only five years, the percentage of all adults protected gradually drops. As a result the program has larger impact in the first few years than in the later years.

Although the cohort vaccination program shows little effect this is due to the assumption of a vaccine of 5 years duration and the assignment of risk behavior categories to individuals at age 15 . A vaccine with life time duration would have a much larger impact in the long term. In fact, it would eventually produce greater impact than the standard 
Figure 17. Effects of campaign type on HIV incidence in rural Zimbabwe. "Cohort" vaccinates only 15 -year olds.

"Cohort lifetime" uses a vaccine with lifetime protection. "Catch-up and cohort" vaccinates adults in the first five years, then 15-year olds.

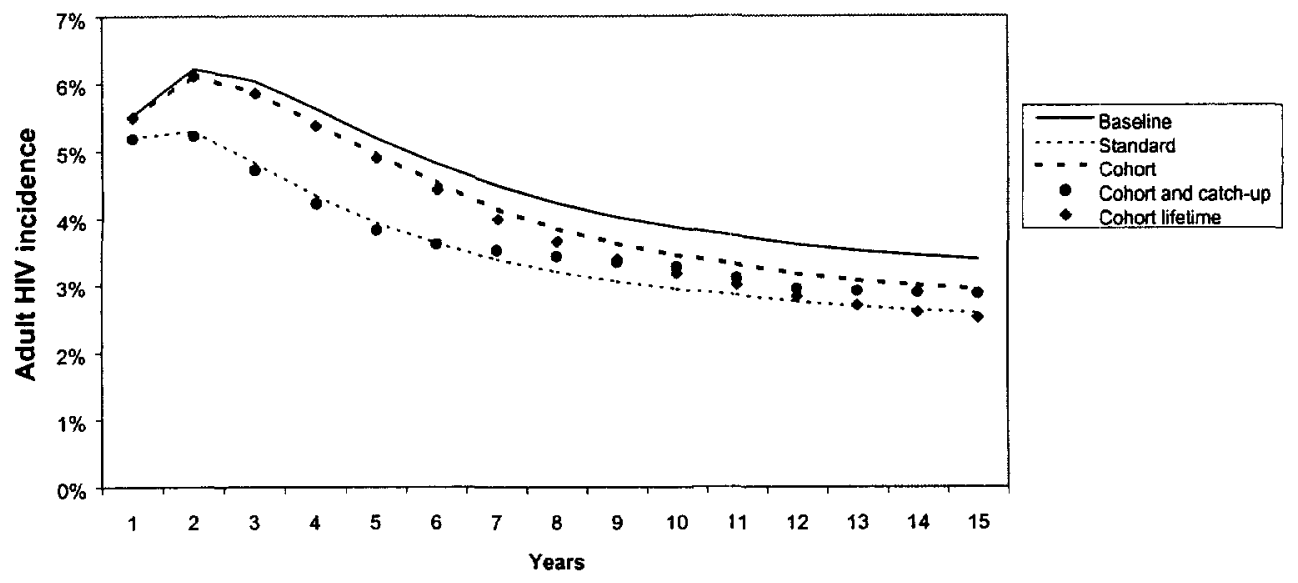

case, since the proportion of all adults protected by vaccination would eventually exceed 65 percent as those who are not protected die at a higher rate. Only blanket vaccination in the first few years of the program will provide a large immediate impact, however.

\section{Behavioral response}

If people who have adopted safer sex practices in the past few years revert back to riskier behavior because they feel protected by a vaccine, the positive effects of the vaccine will be reduced. If this behavior reversal takes place only among those who are vaccinated it is not likely to offset all the gains from the vaccine. If riskier behavior is adopted by even those who are not vaccinated, in the belief that the vaccinations of others will protect them as well, then the net impact of the vaccination program could be negative. This is illustrated in Figure 18. 
Figure 18. Effects on HIV prevalence of behavioral reversals among those vaccinated and all adults in rural

Zimbabwe

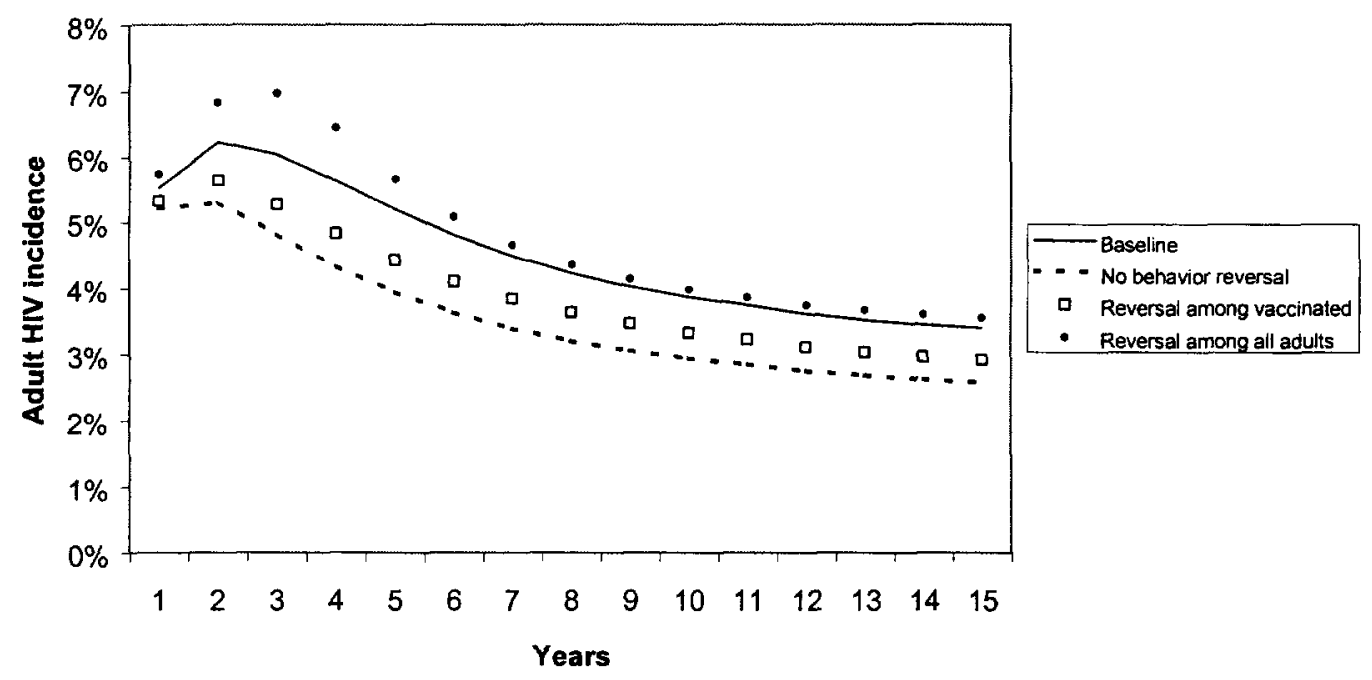

In Kampala and Thailand, significant behavior change has led to declining prevalence. In these settings also, behavioral reversal can eliminate much of the benefits of vaccination as shown in Figure 19.

Figure 19. Effects on HIV incidence of behavioral reversals among those vaccinated and all adults in Kampala and Thailand
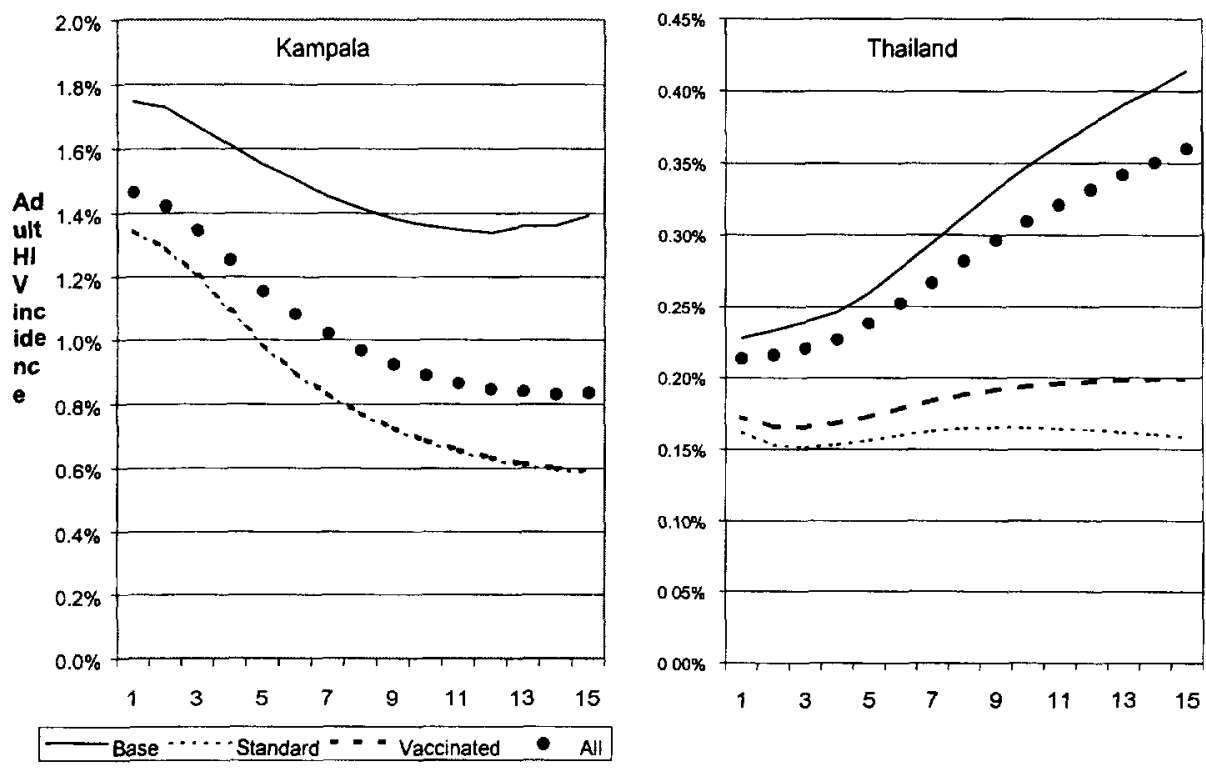
The net effect of behavioral reversals will depend heavily on vaccine efficacy, duration, program strategy and the behavioral response. If efficacy is low and duration is short or programs such as cohort vaccination are used then the impact of the vaccine will be relatively small. In these cases, behavior reversal could eliminate most of the gains. If behavior reversal takes place among everyone, vaccinated or not, then the net impact could be negative. On the other hand, if the efficacy and coverage are high, then behavioral reversals will erode the impact but a substantial benefit will remain.

It is important to note that behavioral reversals among those who are vaccinated will have little effect for a take type vaccine of high efficacy. In the best case of a vaccine that effectively protects for life 95 percent of those vaccinated, no amount of behavior change will result in HIV infection. The effects of behavior reversal will become important only with vaccines of lower efficacy or short duration.

\section{Comparison with other prevention interventions}

Vaccination programs may have to compete for funding with programs designed to increase condom use, treat sexually transmitted infections and reduce the number of partners. We simulated the impact of three alternative interventions for comparison with the "standard" vaccination program of 65 percent coverage of all adults with a 50 percent degree-type efficacy vaccine:

- Condoms: Condom use increases to 70 percent in single high-risk groups and 60 percent in paired high-risk groups over a five-year period and then maintained at those levels.

- Partner reduction: Concurrent partners decrease by 85 percent. (Concurrent partners include polygamous relationships, a long-term partner and a lover, a long-term partner and a casual encounter, or a lover and a casual encounter.)

- STD treatment: The duration of an infectious lesion in high-risk patients is reduced by 80 percent over a five-year period and then maintained at that level.

The results of our simulations indicate that a reasonably effective vaccine is likely to have somewhat greater effect than other preventive interventions. This is illustrated in Figure 20. There are two major reasons for this. First, condom use tends to be inconsistent. People who use condoms often use them selectively with certain partners and in certain situations. The protection may not cover all acts of sexual intercourse as vaccine protection does. Second, abstinence or reductions in the number of partners are not easy to promote. It will generally be easier to reach high coverage levels with a vaccination program than to reach similar levels of protection through behavior change programs. Of course, the best programs will be those that combine vaccination with other prevention interventions. 
Figure 20. Effects on HIV incidence of the standard vaccine compared to other prevention interventions
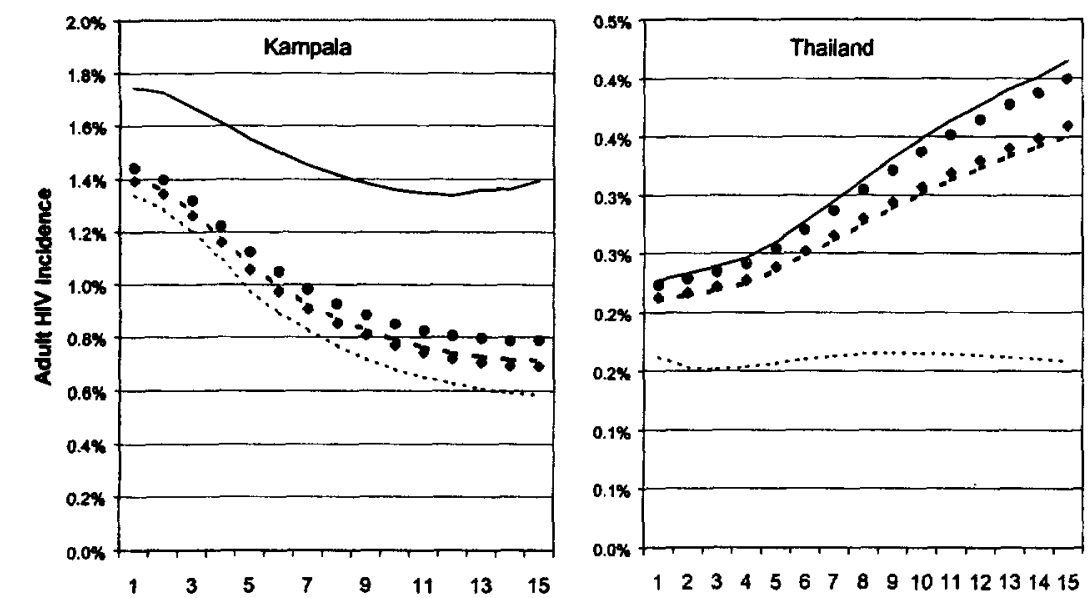

- Base $\cdots \cdot \cdot$ Stendard $\cdots$ Condoms $\bullet$ STDs $\bullet$ Partner reduction

In the standard vaccine program with a vaccine cost of $\$ 20$ per person vaccinated, the cost to avert one new infection is $\$ 210-\$ 1410$. This is comparable to the costs found for other interventions, as shown in Table 5. (Note that the results shown in Table 5 are for selected interventions, countries, and years where cost-effectiveness analysis has been estimated. These results may not apply to other countries or time periods.)

Table 5. Cost per infection averted for different prevention programs

\begin{tabular}{|c|c|c|}
\hline Intervention & $\begin{array}{l}\text { Cost per HIV infection } \\
\text { averted (US Dollars) }\end{array}$ & Country \\
\hline AIDS vaccine ${ }^{28}$ & $\$ 210-\$ 1410$ & Thailand, Uganda, rural Zimbabwe \\
\hline Syndromic management of STIs ${ }^{29}$ & $\$ 217$ & Tanzania \\
\hline Safe blood supply ${ }^{30}$ & $\$ 172$ & Uganda \\
\hline Voluntary counseling and testing ${ }^{31}$ & $\$ 241-\$ 303$ & Kenya, Tanzania \\
\hline $\begin{array}{l}\text { Prevention of mother to child } \\
\text { transmission }^{32}\end{array}$ & $\$ 298-\$ 1265$ & Sub-Saharan Africa \\
\hline
\end{tabular}

\footnotetext{
${ }^{28}$ Assumes vaccination cost of $\$ 20$ /person, 50 percent effective degree type vaccine with 65 percent coverage of adults.

${ }^{29}$ Kathy Attawell and Heiner Grosskurth. 1999. From knowledge to practice: STD control and HIV prevention. European Commission, Luxembourg.

${ }^{30}$ Rex Winsbury 1995. Safe Blood in Developing Countries: The Lessons from Uganda. Luxembourg, European Commission, as reported in Confronting AIDS: Public Priorities in a Global Epidemic Washington, DC: Oxford University Press, 1997.

${ }^{31}$ Michael Sweat, Steven Grigorich, Gloria Sangiwa, Colin Furlonge, Donald Balmer, Claudes Kamenga, Olga Grinstead, Thomas Coates. 2000. "Cost-effectiveness of voluntary HIV-1 counseling and testing in reducing sexual transmission of HIV-1 in Kenya and Tanzania." The Lancet 356: 113-21.
} 
Although very effective condom promotion or partner change programs could have more impact than vaccines of low efficacy or short duration administered to few people, in general we expect that vaccine programs will prove to be as effective or more effective than other prevention interventions. However, only the most effective, long-lasting vaccine reduces HIV incidence to very low levels. Therefore, any future vaccine programs should be combined with other behavior interventions in order to produce the maximum progress against the AIDS epidemic.

\section{Conclusions}

The ideal AIDS vaccine would be inexpensive and have very high efficacy with lifetime duration. It would be implemented through a program that reaches most of the adult population and would be supported by a communications program that warns of the dangers of reverting to risky sexual behavior. If such a vaccine were to become available the advantages of supporting rapid implementation of vaccination programs throughout the world would be obvious. Although such a vaccine would not eliminate AIDS from the world, it would provide the means to reduce HIV prevalence to very low endemic levels.

The first AIDS vaccines may not have all these characteristics. A vaccine with low efficacy and short duration could have a negative impact on public health if its implementation were accompanied by widespread reversion to riskier sexual behaviors because of the mistaken belief that vaccination eliminated the threat of HIV infection.

With vaccines between these two extremes, the decision of whether or not to implement widespread vaccination programs is less clear. In this report we have used simulation modeling to examine many of the questions that are likely to arise in the near future as the first wave of vaccine trials starts to produce results.

High efficacy is clearly desirable, but vaccines with efficacies as low as 50 percent can still be quite useful in controlling the epidemic if coverage is high (65 percent of adults). However, with low efficacy vaccines it will be very important to support the vaccination program with efforts to combat any reversal to riskier sex. If efforts to maintain safer sex behaviors are not successful, then behavioral reversals could eliminate most of the benefits of the vaccine. In some cases, the effect could be to increase HIV incidence.

A vaccine with lifetime protection would be ideal. However, vaccines with shorter durations of effectiveness could still be useful, especially if they are used in programs involving regular re-vaccination. Of course, the re-vaccination program will add to the costs. For many people, the period of highest risk of HIV infection is the period between the initiation of sexual activity and the formation of a stable union. For these people, vaccination before the start of sexual activity with a vaccine that is effective for only a few years could still provide substantial benefits even if no re-vaccination takes place.

\footnotetext{
${ }^{32}$ Elliot Marseille, James G. Khan, Francis Mmiro, Laura Guay, Philippa Musoke, Lary Glenn Fowler, J. Brooks Jackson. 1999. "Cost effectiveness of single-dose nevirapine regimen for mothers and babies to decrease vertical HIV-1 transmission in Sub-Saharan Africa." The Lancet 354(9181): 803-09.
} 
If vaccine purchase and delivery is very expensive, it may be useful to think about concentrating public subsidies on vaccinating certain target groups rather than the entire population. If vaccinations are targeted to high risk groups, such as those engaging in commercial sex or adolescents, the cost-effectiveness may be substantially better than for programs vaccinating all adults, but the total impact may be less. While such programs might be of use in countries where HIV infection is limited to certain risk groups, they might be politically difficult to justify in countries with generalized epidemics.

Even the most effective vaccine programs will not completely eliminate HIV infections. Other prevention programs should continue in conjunction with vaccination programs in order to reduce HIV infections to the lowest possible levels and maintain the other health benefits, such as prevention of sexually transmitted diseases. 




\section{Policy Research Working Paper Series}

Title

WPS2791 The Static and Dynamic Incidence of Vietnam's Public Safety Net

WPS2792 Determinants of Life Insurance Consumption across Countries

WPS2793 Agricultural Markets and Risks: Management of the Latter, Not the Former

WPS2794 Land Policies and Evolving Farm Structures in Transition Countries
Author

Dominique van de Walle

Thorsten Beck

lan Webb

Panos Varangis

Donald Larson

Jock R. Anderson

Zvi Lerman

Csaba Csaki

Gershon Feder

Adam Wagstaff

WPS2795 Inequalities in Health in Developing Countries: Swimming against the Tide?

WPS2796 Do Rural Infrastructure Investments Jocelyn A. Songco Benefit the Poor? Evaluating Linkages:

A Global View, A Focus on Vietnam

WPS2797 Regional Integration and Development Maurice Schiff in Small States

WPS2798 Fever and Its Treatment among the More or Less Poor in Sub-Saharan Africa

WPS2799 The Impact of the Indonesian Financial Crisis on Children: Data from 100 Villages Survey

WPS2800 Did Social Safety Net Scholarships Reduce Drop-Out Rates during the Indonesian Economic Crisis?

WPS2801 Policies to Promote Saving for Retirement: A Synthetic Overview

WPS2802 Telecommunication Reforms, Access Regulation, and Internet Adoption in Latin America

Deon Filmer

Lisa A. Cameron

Lisa A. Cameron

Dimitri Vittas

Antonio Estache Marco Manacorda Tommaso M. Valletti

WPS2803 Determinants of Agricultural Growth in Indonesia, the Philippines, and Thailand

Yair Mundlak Donald F. Larson Rita Butzer

WPS2804 Liberalizing Trade in Agriculture: Developing Countries in Asia and the John S. Wilson Post-Doha Agenda

WPS2805 To Spray or Not to Spray? Pesticides, John S. Wilson Banana Exports, and Food Safety Tsunehiro Otsuki
Date

Contact for paper

February 2002

H. Sladovich 37698

February 2002

A. Yaptenco 31823

February 2002

P. Kokila 33716

February 2002

M. Fernandez 33766

February 2002

H. Sladovich 37698

February 2002

H. Sutrisna 88032

February 2002

March 2002

March 2002

March 2002

P. Sader 33902

March 2002

P. Infante 37642

March 2002

March 2002

G. Chenet-Smith 36370

P. Kokila 33716

March 2002

P. Flewitt 32724

March 2002
P. Flewitt 32724 


\section{Policy Research Working Paper Series}

Title

WPS2806 $\begin{aligned} & \text { Dirty Exports and Environmental } \\ & \text { Regulation: Do Standards Matter to } \\ & \text { Trade? }\end{aligned}$
WPS 2807 The Role of Natural Resources in
Fundamental Tax Reform in the
Russian Federation

Author

John S. Wilson

Tsunehiro Otsuki

Benoît Bosquet

Andrew Powell

Robert D. Ebel

Serdar Yilmaz

Luc Christiaensen

Lionel Demery

Stefano Paternostro
Date

March 2002

March 2002

March 2002

March 2002

March 2002
Contact for paper

P. Flewitt 32724

D. Duff 39506

E. Mekhova 85984

M. Morris 37285

N. Nouviale 34514 